\title{
New Approaches to Polysubstituted Pyrroles and $\gamma$-Lactams based on Nucleophilic Addition of Ti(IV) Enolates derived from $\alpha$-Diazo- $\beta$-keto Carbonyl Compounds to $N$-Tosylimines
}

Changqing Dong, Guisheng Deng and Jianbo Wang*

Beijing National Laboratory of Molecular Sciences (BNLMS), Key Laboratory of Bioorganic Chemistry and Molecular Engineering of Ministry of Education, Department of Chemical Biology, College of Chemistry, Peking University, Beijing 100871, China; wangjb@pku.edu.cn

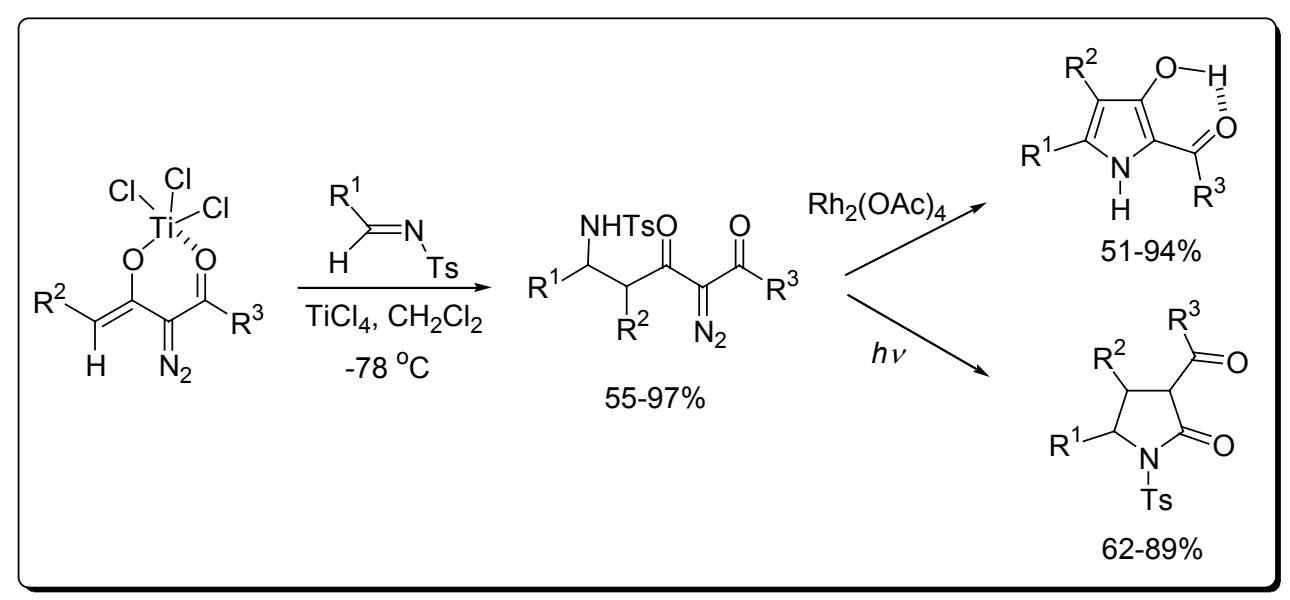

\section{CONTENTS}

Spectra data for $\mathbf{3 a} \sim \mathbf{v}$

Spectra data for $\mathbf{6} \mathbf{a} \sim \mathbf{n}$

Spectra data for $\mathbf{7 a} \sim \mathbf{m}$

${ }^{1} \mathrm{H}$ and ${ }^{13} \mathrm{C}$ NMR for $7 \mathbf{i}$.

${ }^{1} \mathrm{H}$ and ${ }^{13} \mathrm{C}$ NMR for $7 \mathbf{k}$ 
Ethyl 2-Diazo-3-oxo-5-( $\mathbf{N}$-tosyl)amino-5-phenylpentanoate (3a). mp $140-142{ }^{\circ} \mathrm{C}$; IR ( $\mathrm{KBr}$ ) 3223, 2152, 1748, $1625 \mathrm{~cm}^{-1}$; ${ }^{1} \mathrm{H}$ NMR $\left(200 \mathrm{MHz}, \mathrm{CDCl}_{3}\right) \delta 1.32(\mathrm{t}, J=7.2 \mathrm{~Hz}, 3 \mathrm{H}), 2.37(\mathrm{~s}, 3 \mathrm{H})$, $3.19(\mathrm{dd}, J=15.6,5.4 \mathrm{~Hz}, 1 \mathrm{H}), 3.36(\mathrm{dd}, J=15.6,8.0 \mathrm{~Hz}, 1 \mathrm{H}), 4.28(\mathrm{q}, J=7.2 \mathrm{~Hz}, 2 \mathrm{H})$, 4.76-4.86 (m, 1H), $5.69(\mathrm{~d}, J=7.8 \mathrm{~Hz}, 1 \mathrm{H}), 7.14-7.20(\mathrm{~m}, 7 \mathrm{H}), 7.58(\mathrm{~d}, J=8.2 \mathrm{~Hz}, 2 \mathrm{H}) ;{ }^{13} \mathrm{C}$ NMR $\left(50 \mathrm{MHz}, \mathrm{CDCl}_{3}\right) \delta 14.2,21.4,46.3,54.5,61.6,76.2,126.3,127.0,127.4,128.4,129.2$, 137.4, 140.1, 143.0, 161.1, 189.8; MS m/z (FAB) $416\left[(\mathrm{M}+\mathrm{H})^{+}, 13\right], 388$ (3), 344 (2), 261 (11), 245 (69), 219 (20), 181(12), 171 (49), 139 (23), 115 (38), 91 (100), 77 (22), 59 (38), 41 (53). Anal. Calcd for $\mathrm{C}_{20} \mathrm{H}_{21} \mathrm{~N}_{3} \mathrm{O}_{5} \mathrm{~S}: \mathrm{C}, 57.82 ; \mathrm{H}, 5.09 ; \mathrm{N}, 10.11$. Found: C, 57.85; H, 5.09; N, 10.01 .

Ethyl 2-Diazo-3-oxo-5-( $N$-tosyl)amino-5-(2-methylphenyl)pentanoate (3b). mp $112-114{ }^{\circ} \mathrm{C}$; IR (KBr) 3212, 2151, 1715, $1626 \mathrm{~cm}^{-1} ;{ }^{1} \mathrm{H}$ NMR $\left(200 \mathrm{MHz}, \mathrm{CDCl}_{3}\right) \delta 1.31(\mathrm{t}, J=7.2 \mathrm{~Hz}, 3 \mathrm{H})$, $2.26(\mathrm{~s}, 3 \mathrm{H}), 2.34(\mathrm{~s}, 3 \mathrm{H}), 3.17(\mathrm{dd}, J=15.6,5.6 \mathrm{~Hz}, 1 \mathrm{H}), 3.38(\mathrm{dd}, J=15.6,8.0 \mathrm{~Hz}, 1 \mathrm{H}), 4.28(\mathrm{q}$, $J=7.2 \mathrm{~Hz}, 2 \mathrm{H}), 4.76-4.86(\mathrm{~m}, 1 \mathrm{H}), 5.69$ (d, $J=7.8 \mathrm{~Hz}, 1 \mathrm{H}), 7.02-7.18(\mathrm{~m}, 6 \mathrm{H}), 7.53(\mathrm{~d}, J=8.2$ $\mathrm{Hz}, 2 \mathrm{H}) ;{ }^{13} \mathrm{C}$ NMR $\left(50 \mathrm{MHz}, \mathrm{CDCl}_{3}\right) \delta 14.1,18.8,21.2,45.5,50.6,61.5,76.6,126.0,126.7$, $127.1,129.0,130.1,134.8,137.3,138.2,142.7,161.0,189.6$; MS $m / z(\mathrm{FAB}) 430\left[(\mathrm{M}+\mathrm{H})^{+}, 16\right]$, 402 (21), 274 (99), 259 (62), 185 (71), 155 (97), 129 (48), 118 (55), 91 (100), 77 (46), 45 (66). Anal. Calcd for $\mathrm{C}_{21} \mathrm{H}_{23} \mathrm{~N}_{3} \mathrm{O}_{5} \mathrm{~S}$ : C, 58.73; H, 5.40; N, 9.78. Found: C, 58.63; H, 5.43; N, 9.67.

Ethyl 2-Diazo-3-oxo-5-( $\mathrm{N}$-tosyl)amino-5-(4-fluorophenyl)pentanoate (3c). mp $143{ }^{\circ} \mathrm{C}$ (dec.); IR (KBr) 3235, 2146, 1708, $1629 \mathrm{~cm}^{-1} ;{ }^{1} \mathrm{H}$ NMR $\left(200 \mathrm{MHz}, \mathrm{CDCl}_{3}\right) \delta 1.32(\mathrm{t}, J=7.2 \mathrm{~Hz}, 3 \mathrm{H})$, $2.38(\mathrm{~s}, 3 \mathrm{H}), 3.18(\mathrm{dd}, J=15.4,5.4 \mathrm{~Hz}, 1 \mathrm{H}), 3.30(\mathrm{dd}, J=15.5,7.7 \mathrm{~Hz}, 1 \mathrm{H}), 4.28(\mathrm{q}, J=7.2 \mathrm{~Hz}$, 2H), 4.74-4.85 (m, 1H), $5.81(\mathrm{~d}, J=7.4 \mathrm{~Hz}, 1 \mathrm{H}), 6.87(\mathrm{t}, J=8.7 \mathrm{~Hz}, 2 \mathrm{H}), 7.14-7.21(\mathrm{~m}, 4 \mathrm{H})$, $7.57(\mathrm{~d}, J=8.6 \mathrm{~Hz}, 2 \mathrm{H}) ;{ }^{13} \mathrm{C}$ NMR $\left(50 \mathrm{MHz}, \mathrm{CDCl}_{3}\right) \delta 14.3,21.4,46.2,54.0,61.8,115.0,115.5$, $127.1,128.1,128.3,129.3,136.0,137.5,143.2,159.6,161.1,164.5,189.8 ; \mathrm{MS} m / z$ (FAB) 434 $\left[(\mathrm{M}+\mathrm{H})^{+}, 4\right], 263$ (60), 251 (4), 237 (10), 204 (7), 189 (43), 156 (49), 139 (29), 124 (26), 91 (100), 77 (18), 45 (68). Anal. Calcd for $\mathrm{C}_{20} \mathrm{H}_{20} \mathrm{FN}_{3} \mathrm{O}_{5} \mathrm{~S}: \mathrm{C}, 55.42 ; \mathrm{H}, 4.65 ; \mathrm{N}, 9.69$. Found: C, 55.44; $\mathrm{H}$, $4.60 ; \mathrm{N}, 9.68$.

Ethyl 2-Diazo-3-oxo-5-( $N$-tosyl)amino-5-(4-chlorophenyl)pentanoate (3d). mp $149-152{ }^{\circ} \mathrm{C}$ (dec.); IR (KBr) 3214, 2157, 1719, $1625 \mathrm{~cm}^{-1} ;{ }^{1} \mathrm{H}$ NMR (200 MHz, $\left.\mathrm{CDCl}_{3}\right) \delta 1.32$ (t, $J=7 \mathrm{~Hz}$, $3 \mathrm{H}), 2.39(\mathrm{~s}, 3 \mathrm{H}), 3.17(\mathrm{dd}, J=15.6,5.4 \mathrm{~Hz}, 1 \mathrm{H}), 3.29(\mathrm{dd}, J=15.6,7.8 \mathrm{~Hz}, 1 \mathrm{H}), 4.28(\mathrm{q}, J=7.0$ $\mathrm{Hz}, 2 \mathrm{H}), 4.73-4.83(\mathrm{~m}, 1 \mathrm{H}), 5.85(\mathrm{~d}, J=7.8 \mathrm{~Hz}, 1 \mathrm{H}), 7.15-7.19(\mathrm{~m}, 6 \mathrm{H}), 7.56(\mathrm{~d}, J=8.2 \mathrm{~Hz}, 2 \mathrm{H})$; ${ }^{13} \mathrm{C}$ NMR $\left(50 \mathrm{MHz}, \mathrm{CDCl}_{3}\right) \delta 14.3,21.4,46.0,54.0,61.8,127.1,127.9,128.5,129.3,133.3$, 137.3, 138.6, 143.3, 161.1, 189.7; MS m/z (FAB) $450\left[(\mathrm{M}+\mathrm{H})^{+}, 7\right], 422$ (3), 378 (2), 294 (52), 279 (65), 253 (19), 237 (2), 222 (12), 205 (50), 192 (7), 179 (16), 155 (87), 139 (63), 125 (12), 115 (35), 91 (100), 77 (33), 59 (48), 45 (80). Anal. Calcd for $\mathrm{C}_{20} \mathrm{H}_{20} \mathrm{ClN}_{3} \mathrm{O}_{5} \mathrm{~S}: \mathrm{C}, 53.39 ; \mathrm{H}, 4.48$; N, 9.34. Found: C, 53.53; H, 4.48; N, 9.12 .

Ethyl 2-Diazo-3-oxo-5-( $\boldsymbol{N}$-tosyl)amino-5-(3-cyanophenyl)pentanoate (3e). mp $120-121{ }^{\circ} \mathrm{C}$; IR (KBr) 3193, 2232, 2157, 1714, $1619 \mathrm{~cm}^{-1} ;{ }^{1} \mathrm{H}$ NMR $\left(200 \mathrm{MHz}, \mathrm{CDCl}_{3}\right) \delta 1.33(\mathrm{t}, J=7.2 \mathrm{~Hz}$, $3 \mathrm{H}), 2.40(\mathrm{~s}, 3 \mathrm{H}), 3.24(\mathrm{~d}, J=6.4 \mathrm{~Hz}, 2 \mathrm{H}), 4.29$ (q, $J=7.2 \mathrm{~Hz}, 2 \mathrm{H}), 4.79-4.89(\mathrm{~m}, 1 \mathrm{H}), 6.03$ (d, $J$ 
$=7.2 \mathrm{~Hz}, 1 \mathrm{H}), 7.18(\mathrm{~d}, J=8.2 \mathrm{~Hz}, 2 \mathrm{H}), 7.27-7.58(\mathrm{~m}, 6 \mathrm{H}) ;{ }^{13} \mathrm{C} \mathrm{NMR}\left(50 \mathrm{MHz}, \mathrm{CDCl}_{3}\right) \delta 14.2$, 21.4, 46.1, 53.8, 61.9, 76.8, 112.3, 118.4, 127.0, 129.2, 129.4, 131.0, 131.2, 137.1, 141.7, 143.5, 161.0, 189.3; MS m/z (FAB) 441[(M+H) +3$], 413$ (3), 285 (16), 270 (6), 258 (4), 196 (14), 181 (3), 155 (57), 139 (29), 115 (8), 91 (100), 77 (21), 45 (65). Anal. Calcd for $\mathrm{C}_{21} \mathrm{H}_{20} \mathrm{~N}_{4} \mathrm{O}_{5} \mathrm{~S}$ : C, 57.26; H, 4.58; N, 12.72. Found: C, 57.13; H, 4.61; N, 12.70 .

Ethyl 2-Diazo-3-oxo-5-( $\mathrm{N}$-tosyl)amino-5-(4-methoxyphenyl)pentanoate (3f). mp 107-110 ${ }^{\circ} \mathrm{C}$; IR (KBr) 3236, 2361, 2143, 1713, $1626 \mathrm{~cm}^{-1} ;{ }^{1} \mathrm{H}$ NMR $\left(200 \mathrm{MHz}, \mathrm{CDCl}_{3}\right) \delta 1.32(\mathrm{t}, J=7 \mathrm{~Hz}$, $3 \mathrm{H}), 2.38(\mathrm{~s}, 3 \mathrm{H}), 3.19(\mathrm{dd}, J=15.7,5.5 \mathrm{~Hz}, 1 \mathrm{H}), 3.34(\mathrm{dd}, J=15.7,7.8 \mathrm{~Hz}, 1 \mathrm{H}), 3.75(\mathrm{~s}, 3 \mathrm{H})$, $4.28(\mathrm{q}, J=7 \mathrm{~Hz}, 2 \mathrm{H}), 4.70-4.81(\mathrm{~m}, 1 \mathrm{H}), 5.59(\mathrm{~d}, J=7.6 \mathrm{~Hz}, 1 \mathrm{H}), 6.72(\mathrm{~d}, J=8.4 \mathrm{~Hz}, 2 \mathrm{H}), 7.10$ $(\mathrm{d}, J=8.4 \mathrm{~Hz}, 2 \mathrm{H}), 7.17(\mathrm{~d}, J=8.3 \mathrm{~Hz}, 2 \mathrm{H}), 7.58(\mathrm{~d}, J=8.3 \mathrm{~Hz}, 2 \mathrm{H}) ;{ }^{13} \mathrm{C} \mathrm{NMR}\left(50 \mathrm{MHz}, \mathrm{CDCl}_{3}\right)$ $\delta 14.2,21.3,46.3,54.1,55.1,61.6,76.8,113.7,127.0,127.6,129.2,132.2,137.5,142.9,158.8$, 161.0, 189.9; MS m/z (FAB) 452 [(M+Li) $\left.{ }^{+}, 20\right], 275$ (56), 249 (11), 201 (27), 155 (23), 91 (100), 47 (66). Anal. Calcd for $\mathrm{C}_{21} \mathrm{H}_{23} \mathrm{~N}_{3} \mathrm{O}_{6} \mathrm{~S}$ : C, 56.62; H, 5.20; N, 9.43. Found: C, 56.61; H, 5.09; N, 9.49 .

Ethyl 2-Diazo-3-oxo-5-( $N$-tosyl)amino-5-[(E)-cinnamyl]pentanoate (3h). mp 99-101 ${ }^{\circ} \mathrm{C}$; IR (KBr) 3231, 2361, 2152, 1710, $1653 \mathrm{~cm}^{-1} ;{ }^{1} \mathrm{H} \mathrm{NMR}\left(200 \mathrm{MHz}, \mathrm{CDCl}_{3}\right) \delta 1.26(\mathrm{t}, J=7.2 \mathrm{~Hz}, 3 \mathrm{H})$, 2.23 (s, 3H), 3.15-3.19 (m, 2H), $4.23(\mathrm{q}, J=7.2 \mathrm{~Hz}, 2 \mathrm{H}), 4.35-4.50(\mathrm{~m}, 1 \mathrm{H}), 5.87-5.99(\mathrm{~m}, 2 \mathrm{H})$, $6.28(\mathrm{~d}, J=16.2 \mathrm{~Hz}, 1 \mathrm{H}), 7.07-7.25(\mathrm{~m}, 7 \mathrm{H}), 7.72(\mathrm{~d}, J=8.2 \mathrm{~Hz}, 2 \mathrm{H}) ;{ }^{13} \mathrm{C} \mathrm{NMR}(50 \mathrm{MHz}$, $\left.\mathrm{CDCl}_{3}\right) \delta 13.9,20.9,44.8,52.5,61.3,76.4,126.0,126.9,127.3,127.5,127.9,129.1,131.1,135.7$, 137.6, 142.8, 160.7, 189.7; MS m/z (FAB) 448 [(M+Li) $\left.{ }^{+}, 6\right], 420$ (2), 286 (6), 266 (12), 169 (10), 115 (47), 91 (100), 45 (36). Anal. Calcd for $\mathrm{C}_{22} \mathrm{H}_{23} \mathrm{~N}_{3} \mathrm{O}_{5} \mathrm{~S}$ : C, 59.85; H, 5.25; N, 9.52. Found: C, $59.61 ; \mathrm{H}, 5.27 ; \mathrm{N}, 9.42$.

Ethyl 2-Diazo-3-oxo-5-( $N$-tosyl)amino-5-(2-furyl)pentanoate (3i). mp 120-122 ${ }^{\circ} \mathrm{C}$; IR ( $\mathrm{KBr}$ ) 3259, 2140, 1710, $1644 \mathrm{~cm}^{-1}$; ${ }^{1} \mathrm{H} \mathrm{NMR}\left(200 \mathrm{MHz}, \mathrm{CDCl}_{3}\right) \delta 1.33(\mathrm{t}, J=7.2 \mathrm{~Hz}, 3 \mathrm{H}), 2.43(\mathrm{~s}, 3 \mathrm{H})$, $3.21(\mathrm{dd}, J=16.5,5.7 \mathrm{~Hz}, 1 \mathrm{H}), 3.53(\mathrm{dd}, J=16.5,6.6 \mathrm{~Hz}, 1 \mathrm{H}), 4.29$ (q, $J=7.2 \mathrm{~Hz}, 2 \mathrm{H})$, 4.87-4.98 (m, 1H), $5.61(\mathrm{~d}, J=9 \mathrm{~Hz}, 1 \mathrm{H}), 6.01-6.03(\mathrm{~m}, 1 \mathrm{H}), 6.15-6.18(\mathrm{~m}, 1 \mathrm{H}), 7.16-7.18(\mathrm{~m}$, $1 \mathrm{H}), 7.22(\mathrm{~d}, J=8.0 \mathrm{~Hz}, 2 \mathrm{H}), 7.65(\mathrm{~d}, J=8.0 \mathrm{~Hz}, 2 \mathrm{H}) ;{ }^{13} \mathrm{C} \mathrm{NMR}\left(50 \mathrm{MHz}, \mathrm{CDCl}_{3}\right) \delta 14.0,21.1$, 43.4, 48.0, 61.4, 76.5, 106.7, 110.0, 126.7, 129.1, 137.4, 141.5, 142.8, 152.2, 160.8, 189.0; MS $\mathrm{m} / \mathrm{z}(\mathrm{FAB}) 412$ [(M+Li) $\left.{ }^{+}, 10\right], 386$ (9), 340 (4), 265 (10), 256 (7), 229 (25), 205 (11), 184 (7), 157 (24), 115 (100), 91 (17), 81 (8), 47 (25), 43 (13). Anal. Calcd for $\mathrm{C}_{18} \mathrm{H}_{19} \mathrm{~N}_{3} \mathrm{O}_{6} \mathrm{~S}: \mathrm{C}, 53.33 ; \mathrm{H}$, 4.72; N, 10.36. Found: C, 53.41; H, 4.64; N, 10.20 .

2-Diazo-1,5-diphenyl-5-( $\boldsymbol{N}$-tosyl)amino-1,3-pentanedione (3j). oil; IR (film) 3276, 2125, $1642 \mathrm{~cm}^{-1} ;{ }^{1} \mathrm{H}$ NMR $\left(300 \mathrm{MHz}, \mathrm{CDCl}_{3}\right) \delta 2.30(\mathrm{~s}, 3 \mathrm{H}), 3.32(\mathrm{dd}, J=12.0,5.4 \mathrm{~Hz}, 1 \mathrm{H}), 3.46(\mathrm{dd}$, $J=12.0,8.4 \mathrm{~Hz}, 1 \mathrm{H}), 4.93 \sim 4.91(\mathrm{~m}, 2 \mathrm{H}), 6.02(\mathrm{~d}, J=8.1 \mathrm{~Hz}, 1 \mathrm{H}), 7.27 \sim 7.11(\mathrm{~m}, 7 \mathrm{H}), 7.62 \sim 7.46$ $(\mathrm{m}, 7 \mathrm{H}) ;{ }^{13} \mathrm{C}$ NMR $\left(75 \mathrm{MHz}, \mathrm{CDCl}_{3}\right) \delta 21.3,47.3,54.8,126.4,127.0,127.3,127.4,128.4,128.9$, $129.2,132.9,136.8,137.5,140.1,142.9,185.1,190.7$; EI-MS $(m / z$, relative intensity): 419 
$\left[(\mathrm{M}-28)^{+}, 6.9\right], 264$ (38), 155 (21), 105 (100), 91 (69), 77 (51). Anal. Calcd for $\mathrm{C}_{24} \mathrm{H}_{21} \mathrm{~N}_{3} \mathrm{O}_{4} \mathrm{~S}$ : C, 64.41; H, 4.73; N, 9.39. Found: C, 64.47; H, 4.64; N, 9.19.

2-Diazo-5-furyl-1-phenyl-5-( $\boldsymbol{N}$-tosyl)amino-1,3-pentanedione (3k). mp 120-121 ${ }^{\circ} \mathrm{C}$; IR ( $\mathrm{KBr}$ ) $3281,2127,1655,1636 \mathrm{~cm}^{-1} ;{ }^{1} \mathrm{H}$ NMR $\left(200 \mathrm{MHz}, \mathrm{CDCl}_{3}\right) \delta 2.36(\mathrm{~s}, 3 \mathrm{H}), 3.33(\mathrm{dd}, J=16.3,5.2$ $\mathrm{Hz}, 1 \mathrm{H}), 3.63(\mathrm{dd}, J=16.3,7.2 \mathrm{~Hz}, 1 \mathrm{H}), 4.97-5.04(\mathrm{~m}, 1 \mathrm{H}), 5.64-5.71$ (br, m, 1H), 6.04 (d, $J=$ $2.8 \mathrm{~Hz}, 1 \mathrm{H}), 6.16-6.18(\mathrm{~m}, 1 \mathrm{H}), 7.17(\mathrm{~s}, 1 \mathrm{H}), 7.21(\mathrm{~d}, J=8.2 \mathrm{~Hz}, 2 \mathrm{H}), 7.46-7.68(\mathrm{~m}, 7 \mathrm{H}) ;{ }^{13} \mathrm{C}$ NMR $\left(50 \mathrm{MHz}, \mathrm{CDCl}_{3}\right) \delta 21.1,44.5,48.2,83.8,106.7,110.0,126.7,127.1,128.7,129.1,132.6$, 136.6, 137.4, 141.6, 142.8, 152.2, 184.7, 189.9; MS m/z (FAB) 444 [(M+Li) $\left.{ }^{+}, 4\right], 295$ (3), 263 (14), 205 (12), 157 (9), 115 (100), 91 (40), 57 (21), 47 (31). Anal. Calcd for $\mathrm{C}_{22} \mathrm{H}_{19} \mathrm{~N}_{3} \mathrm{O}_{5} \mathrm{~S}: \mathrm{C}$, $60.40 ; \mathrm{H}, 4.38 ; \mathrm{N}, 9.61$. Found: C, 60.39; H, 4.32; N, 9.43.

(E)-2-Diazo-1,7-diphenyl-5-( $N$-tosyl)aminohept-6-en-1,3-dione $\quad$ (3I). $\quad$ mp $\quad 78-82 \quad{ }^{\circ} \mathrm{C}$ (decomposed); IR (KBr) 3255, 2240, 1661, $1630 \mathrm{~cm}^{-1} ;{ }^{1} \mathrm{H}$ NMR (200 MHz, $\left.\mathrm{CDCl}_{3}\right) \delta 2.26$ (s, $3 \mathrm{H}), 3.22(\mathrm{dd}, J=16.3,4.9 \mathrm{~Hz}, 1 \mathrm{H}), 3.37(\mathrm{dd}, J=16.3,6.7 \mathrm{~Hz}, 1 \mathrm{H}), 4.39-4.52(\mathrm{~m}, 1 \mathrm{H}), 5.67$ (d, $J=8.8 \mathrm{~Hz}, 1 \mathrm{H}), 5.95(\mathrm{dd}, J=16,7 \mathrm{~Hz}, 1 \mathrm{H}), 6.63(\mathrm{~d}, J=16 \mathrm{~Hz}, 1 \mathrm{H}), 7.11-7.28(\mathrm{~m}, 7 \mathrm{H})$, 7.24-7.62 (m, 5H), $7.72(\mathrm{~d}, J=8.2 \mathrm{~Hz}, 2 \mathrm{H}) ;{ }^{13} \mathrm{C} \mathrm{NMR}\left(50 \mathrm{MHz}, \mathrm{CDCl}_{3}\right) \delta 21.0,46.1,52.9,84.0$, 126.2, 127.0, 127.1, 127.5, 128.1, 128.7, 129.2, 131.3, 132.6, 135.9, 136.7, 137.7, 142.9, 184.8, 190.6; MS m/z (FAB) 480 [(M+Li) $\left.{ }^{+}, 5\right], 452$ (7), 297 (5), 263 (6), 223 (7), 205 (6), 157 (9), 147 (14), 115 (100), 91 (46), 47 (24). Anal. Calcd for $\mathrm{C}_{26} \mathrm{H}_{23} \mathrm{~N}_{3} \mathrm{O}_{4} \mathrm{~S}: \mathrm{C}, 65.95 ; \mathrm{H}, 4.90 ; \mathrm{N}, 8.87$. Found: C, 66.01; H, 4.72; N, 8.91.

3-Diazo-6-phenyl-6-( $\mathbf{N - t o s y l ) a m i n o - 2 , 4 - h e x a n e d i o n e ~ ( 3 m ) . ~ m p ~ 1 3 1 - 1 3 2 ~}{ }^{\circ} \mathrm{C}$; IR (KBr) 3294, 2362, 2132, 1665, $1628 \mathrm{~cm}^{-1}$; ${ }^{1} \mathrm{H}$ NMR $\left(200 \mathrm{MHz}, \mathrm{CDCl}_{3}\right) \delta 2.33(\mathrm{~s}, 3 \mathrm{H}), 2.37(\mathrm{~s}, 3 \mathrm{H}), 3.17$ (dd, $J=15.6,5.5 \mathrm{~Hz}, 1 \mathrm{H}), 3.34(\mathrm{dd}, J=15.6,7.4 \mathrm{~Hz}, 1 \mathrm{H}), 4.76-4.86(\mathrm{~m}, 1 \mathrm{H}), 5.71(\mathrm{~d}, J=7.8 \mathrm{~Hz}, 1 \mathrm{H})$, 7.13-7.17 (m, 7H), $7.57(\mathrm{~d}, J=8.2 \mathrm{~Hz}, 2 \mathrm{H}) ;{ }^{13} \mathrm{C} \mathrm{NMR}\left(50 \mathrm{MHz}, \mathrm{CDCl}_{3}\right) \delta 21.4,28.0,47.1,54.4$, 85.0, 126.4, 127.0, 127.6, 128.5, 129.3, 137.3, 139.8, 143.1, 187.2, 189.1; MS $m / z$ (FAB) 392 $\left[(\mathrm{M}+\mathrm{Li})^{+}, 28\right], 372(14), 366(25), 349$ (14), 315 (12), 307 (32), 285 (4), 265 (18), 247 (2), 223 (28), 205 (10), 189 (4), 157 (27), 121 (39), 115 (100), 91 (10), 59 (9), 47 (24). Anal. Calcd for $\mathrm{C}_{19} \mathrm{H}_{19} \mathrm{~N}_{3} \mathrm{O}_{4} \mathrm{~S}: \mathrm{C}, 59.21 ; \mathrm{H}, 4.97 ; \mathrm{N}, 10.90$. Found: C, 59.01; H, 4.80; N, 10.76.

3-Diazo-6-(4-methoxyphenyl)-6-( $\boldsymbol{N}$-tosyl)amino-2,4-hexanedione (3n). mp 136-138 ${ }^{\circ} \mathrm{C}$; IR (KBr) 3193, 2361, 2150, 1676, $1626 \mathrm{~cm}^{-1} ;{ }^{1} \mathrm{H}$ NMR (200 MHz, $\left.\mathrm{CDCl}_{3}\right) \delta 2.33(\mathrm{~s}, 3 \mathrm{H}), 2.38(\mathrm{~s}$, $3 \mathrm{H}), 3.16(\mathrm{dd}, J=15.5,5.8 \mathrm{~Hz}, 1 \mathrm{H}), 3.31(\mathrm{dd}, J=15.5,7.2 \mathrm{~Hz}, 1 \mathrm{H}), 3.74(\mathrm{~s}, 3 \mathrm{H}), 4.71-4.81$ (m, $1 \mathrm{H}), 5.71(\mathrm{~d}, J=7.4 \mathrm{~Hz}, 1 \mathrm{H}), 6.69(\mathrm{~d}, J=9.6 \mathrm{~Hz}, 2 \mathrm{H}), 6.78(\mathrm{~d}, J=9.6 \mathrm{~Hz}, 2 \mathrm{H}), 7.16(\mathrm{~d}, J=8.4$ $\mathrm{Hz}, 2 \mathrm{H}), 7.57(\mathrm{~d}, J=8.4 \mathrm{~Hz}, 2 \mathrm{H}) ;{ }^{13} \mathrm{C} \mathrm{NMR}\left(50 \mathrm{MHz}, \mathrm{CDCl}_{3}\right) \delta 21.4,28.0,47.1,54.0,55.2,85.0$, $113.8,127.1,127.6,129.3,131.8,137.4,143.0,158.9,187.3,189.1 ; \mathrm{MS} m / z(\mathrm{FAB}) 422\left[(\mathrm{M}+\mathrm{Li})^{+}\right.$, 20], 365 (18), 223 (28), 157 (30), 115 (100), 47 (23), 45 (15). Anal. Calcd for $\mathrm{C}_{20} \mathrm{H}_{21} \mathrm{~N}_{3} \mathrm{O}_{5} \mathrm{~S}$ : C, 57.82; H, 5.09; N, 10.11. Found: C, 57.89; H, 5.03; N, 10.21. 
3-Diazo-6-(3-bromophenyl)-6-( $\boldsymbol{N}$-tosyl)amino-2,4-hexanedione (3o). $\mathrm{mp} \quad 125-127{ }^{\circ} \mathrm{C}$; IR (KBr) 3209, 2151, 1669, $1622 \mathrm{~cm}^{-1}$; ${ }^{1} \mathrm{H}$ NMR $\left(200 \mathrm{MHz}, \mathrm{CDCl}_{3}\right) \delta 2.34$ (s, 3H), 2.38 (s, 3H), $3.16(\mathrm{dd}, J=15.8,5.5 \mathrm{~Hz}, 1 \mathrm{H}), 3.30(\mathrm{dd}, J=15.8,7.6 \mathrm{~Hz}, 1 \mathrm{H}), 4.72-4.83(\mathrm{~m}, 1 \mathrm{H}), 5.74(\mathrm{~d}, J=8$ $\mathrm{Hz}, 1 \mathrm{H}), 7.02-7.30(\mathrm{~m}, 6 \mathrm{H}), 7.54(\mathrm{~d}, J=8.4 \mathrm{~Hz}, 2 \mathrm{H}) ;{ }^{13} \mathrm{C} \mathrm{NMR}\left(50 \mathrm{MHz}, \mathrm{CDCl}_{3}\right) \delta 21.4,27.8$, 46.9, 53.9, 85.1, 122.4, 125.2, 127.0, 129.3, 129.6, 130.0 130.5, 137.2, 142.0, 143.3, 187.0, 188.9; MS m/z (FAB) 472 [(M+Li) $\left.{ }^{+}, 10\right], 444$ (17), 265 (12), 223 (20), 157 (24), 115 (100), 47 (19), 45 (13); Anal. Calcd for $\mathrm{C}_{19} \mathrm{H}_{18} \mathrm{BrN}_{3} \mathrm{O}_{4} \mathrm{~S}: \mathrm{C}, 49.15 ; \mathrm{H}, 3.91$; N, 9.05. Found: $\mathrm{C}, 49.16$; H, 3.87; N, 8.97 .

Ethyl 2-Diazo-4-methyl-3-oxo-5-phenyl-5-( $N$-tosyl)aminopentanoate (3p). The major isomer: IR (film) 3287, 2983, 2140, 1715, 1654, $1160 \mathrm{~cm}^{-1}$; ${ }^{1} \mathrm{H}$ NMR (300 MHz, $\left.\mathrm{CDCl}_{3}\right) \delta 1.01$ (d, $\mathrm{J}=6.9,3 \mathrm{H}), 1.30(\mathrm{t}, J=7.2,3 \mathrm{H}), 2.32(\mathrm{~s}, 3 \mathrm{H}), 4.03-4.23(\mathrm{~m}, 1 \mathrm{H}), 4.27(\mathrm{q}, J=7.2,2 \mathrm{H}), 4.48-4.54$ $(\mathrm{m}, 1 \mathrm{H}), 7.04-7.14(\mathrm{~m}, 7 \mathrm{H}), 7.45-7.48(\mathrm{~m}, 2 \mathrm{H}) ;{ }^{13} \mathrm{C} \mathrm{NMR}\left(75 \mathrm{MHz}, \mathrm{CDCl}_{3}\right) \delta 14.2,15.3,21.3$, 46.1, 61.1, 61.7, 126.7, 127.2, 128.2, 129.0, 137.9, 139.3, 142.6, 161.5, 195.0; EI-MS $(\mathrm{m} / z$, relative intensity): $401\left[(\mathrm{M}-28)^{+}, 5\right], 337$ (33), 260 (65), 155 (57), 91 (100). Anal. Calcd for $\mathrm{C}_{21} \mathrm{H}_{23} \mathrm{~N}_{3} \mathrm{O}_{5} \mathrm{~S}$ : C, 58.73; H, 5.40; N, 9.78. Found: C, 58.72; H, 5.39; N, 9.57.

Ethyl 2-Diazo-4-methyl-5-(4-methoxyphenyl)-3-oxo-5-( $N$-tosyl)aminopentanoate (3q). The major isomer: IR (film) 3278, 2982, 2140, 1715, 1654, $1160 \mathrm{~cm}^{-1} ;{ }^{1} \mathrm{H} \mathrm{NMR}\left(300 \mathrm{MHz}, \mathrm{CDCl}_{3}\right) \delta$ $0.98(\mathrm{~d}, J=6.9 \mathrm{~Hz}, 3 \mathrm{H}), 1.31(\mathrm{t}, J=7.2 \mathrm{~Hz}, 3 \mathrm{H}), 2.33(\mathrm{~s}, 3 \mathrm{H}), 3.74(\mathrm{~s}, 3 \mathrm{H}), 4.00-4.09(\mathrm{~m}, 1 \mathrm{H})$, $4.27(\mathrm{q}, J=7.2 \mathrm{~Hz}, 2 \mathrm{H}), 4.46(\mathrm{t}, J=8.4 \mathrm{~Hz}, 1 \mathrm{H}), 6.02(\mathrm{~d}, J=9.6 \mathrm{~Hz}, 1 \mathrm{H}), 6.65(\mathrm{~d}, J=8.7 \mathrm{~Hz}$, $2 \mathrm{H}), 6.98(\mathrm{~d}, J=8.7 \mathrm{~Hz}, 2 \mathrm{H}), 7.07(\mathrm{~d}, J=8.1 \mathrm{~Hz}, 2 \mathrm{H}), 7.46(\mathrm{~d}, J=8.1 \mathrm{~Hz}, 2 \mathrm{H}) ;{ }^{13} \mathrm{C}$ NMR $(75$ $\left.\mathrm{MHz}, \mathrm{CDCl}_{3}\right) \delta 14.1,15.2,21.3,46.2,55.1,60.6,61.6,113.6,126.7,127.9,129.0,131.5,138.0$, 142.5, 158.7, 161.6, 195.0; EI-MS ( $\mathrm{m} / \mathrm{z}$, relative intensity): $459\left(\mathrm{M}^{+}, 3\right), 431$ (23), 290 (38), 276 (45), 222 (82), 176 (79), 91 (100). Anal. Calcd for $\mathrm{C}_{22} \mathrm{H}_{25} \mathrm{~N}_{3} \mathrm{O}_{6} \mathrm{~S}: \mathrm{C}, 57.50 ; \mathrm{H}, 5.48 ; \mathrm{N}, 9.14$. Found: C, 57.39; H, 5.50; N, 9.09.

Ethyl 2-Diazo-3-oxo-4,5-diphenyl-5-( $N$-tosyl)aminopentanoate (3r). The major isomer: IR (film) $3274,2142,1715,1651 \mathrm{~cm}^{-1} ;{ }^{1} \mathrm{H} \mathrm{NMR}\left(300 \mathrm{MHz}, \mathrm{CDCl}_{3}\right) \delta 1.24(\mathrm{t}, J=7.2 \mathrm{~Hz}, 3 \mathrm{H}), 2.30$ $(\mathrm{s}, 3 \mathrm{H}), 4.14-4.22(\mathrm{~m}, 2 \mathrm{H}), 4.93-4.99(\mathrm{~m}, 1 \mathrm{H}), 5.33-5.39(\mathrm{~m}, 1 \mathrm{H}), 6.09(\mathrm{~d}, J=9.6 \mathrm{~Hz}, 1 \mathrm{H})$, 6.97-7.38 (m, 14H); ${ }^{13} \mathrm{C}$ NMR $\left(75 \mathrm{MHz}, \mathrm{CDCl}_{3}\right) \delta 191.1,160.8,142.7,139.0,137.6,136.6$, $134.4,129.3,129.1,128.3,128.1,128.0,127.5,127.0$; EI-MS $(\mathrm{m} / z$, relative intensity): 463 $\left[(\mathrm{M}-28)^{+}, 6\right], 399$ (61), 308 (64), 260 (44), 176 (100), 131 (57), 91 (50); Anal. Calcd for $\mathrm{C}_{26} \mathrm{H}_{25} \mathrm{~N}_{3} \mathrm{O}_{5} \mathrm{~S}: \mathrm{C}, 63.53 ; \mathrm{H}, 5.13 ; \mathrm{N}, 8.55$. Found: $\mathrm{C}, 63.69 ; \mathrm{H}, 5.35 ; \mathrm{N}, 8.21$.

Ethyl 2-Diazo-5-(2-methylphenyl)-3-oxo-4-phenyl-5-( $N$-tosyl)aminopentanoate (3s). The major isomer: $\mathrm{mp} 130-131{ }^{\circ} \mathrm{C}$; IR (film) 3276, 2982, 2142, 1716, $1650 \mathrm{~cm}^{-1} ;{ }^{1} \mathrm{H}$ NMR $(300 \mathrm{MHz}$, $\left.\mathrm{CDCl}_{3}\right) \delta 1.22(\mathrm{t}, J=7.2 \mathrm{~Hz}, 3 \mathrm{H}), 2.15(\mathrm{~s}, 3 \mathrm{H}), 2.29(\mathrm{~s}, 3 \mathrm{H}), 4.10-4.21(\mathrm{~m}, 2 \mathrm{H}), 5.19-5.25(\mathrm{~m}$, $1 \mathrm{H}), 5.31-5.34(\mathrm{~d}, J=8.1 \mathrm{~Hz}, 1 \mathrm{H}), 6.26(\mathrm{~d}, J=8.7 \mathrm{~Hz}, 1 \mathrm{H}), 6.79-6.82(\mathrm{~m}, 1 \mathrm{H}), 6.92-6.98(\mathrm{~m}$, 4H), 7.10-7.23 (m, 6H), $7.36(\mathrm{~d}, J=8.1 \mathrm{~Hz}, 2 \mathrm{H}) ;{ }^{13} \mathrm{C} \mathrm{NMR}\left(75 \mathrm{MHz}, \mathrm{CDCl}_{3}\right) \delta 14.1,19.1,21.3$, $56.6,56.9,61.5,126.0,126.5,126.6,127.6,128.2$, 128.8, 129.3, 130.0, 134.2, 134.9, 137.1, 
137.6, 142.4, 160.8, 191.2; EI-MS ( $m / z$, relative intensity): $477\left[(\mathrm{M}-28)^{+}, 7\right], 413(13), 274$ (57), 176 (80), 155 (24), 118 (74), 91 (100). Anal. Calcd for $\mathrm{C}_{27} \mathrm{H}_{27} \mathrm{~N}_{3} \mathrm{O}_{5} \mathrm{~S}$ : C, 64.14; H, 5.38; N, 8.31. Found: C, 64.11; H, 5.39; N, 8.08.

The minor product: ${ }^{1} \mathrm{H}$ NMR $\left(300 \mathrm{MHz}, \mathrm{CDCl}_{3}\right) \delta 1.22(\mathrm{t}, J=7.2 \mathrm{~Hz}, 3 \mathrm{H}), 2.34(\mathrm{~s}, 3 \mathrm{H}), 2.47$ (s, $3 \mathrm{H}), 4.15-4.22(\mathrm{~m}, 2 \mathrm{H}), 5.27-5.32(\mathrm{~m}, 1 \mathrm{H}), 5.49(\mathrm{~d}, J=10.8 \mathrm{~Hz}, 1 \mathrm{H}), 6.96-7.04(\mathrm{~m}, 5 \mathrm{H})$, 7.18-7.30 (m, 6H), 7.39-7.41 (m, 2H); ${ }^{13} \mathrm{C}$ NMR $\left(75 \mathrm{MHz}, \mathrm{CDCl}_{3}\right) \delta 14.4,19.4,21.4,29.7$, 57.7, $61.43,125.9,126.9,127.5,128.3,128.9,129.0,129.4,130.4,134.3,136.9,142.5,160.5,189.4$.

Ethyl 5-(4-Chlorophenyl)-2-diazo-3-oxo-4-phenyl-5-( $N$-tosyl)aminopentanoate (3t). The major product: $\mathrm{mp} 136-137{ }^{\circ} \mathrm{C}$; IR (film) $3292,2957,2137,1716,1649 \mathrm{~cm}^{-1} ;{ }^{1} \mathrm{H} \mathrm{NMR}(300 \mathrm{MHz}$, $\left.\mathrm{CDCl}_{3}\right) \delta 1.22(\mathrm{t}, J=7.2,3 \mathrm{H}), 2.40(\mathrm{~s}, 3 \mathrm{H}), 4.72(\mathrm{~d}, J=4.5,1 \mathrm{H}), 4.96-4.91(\mathrm{~m}, 1 \mathrm{H}), 5.30(\mathrm{~d}, J=$ $10.8,1 \mathrm{H}), 7.33 \sim 7.06(\mathrm{~m}, 13 \mathrm{H}) ;{ }^{13} \mathrm{C} \mathrm{NMR}\left(75 \mathrm{MHz}, \mathrm{CDCl}_{3}\right) \delta 14.1,21.5,57.9,58.9,61.5,127.2$, $128.3,128.5,129.1,129.2,129.3,129.6,133.5,136.4,137.5,143.1,160.3,189.2$; EI-MS (m/z, relative intensity): $498\left[(\mathrm{M}-28)^{+}, 5\right], 433$ (8), 342 (32), 294 (22), 176 (100), 131 (64), 91 (46); Anal. Calcd for $\mathrm{C}_{26} \mathrm{H}_{24} \mathrm{ClN}_{3} \mathrm{O}_{5} \mathrm{~S}: \mathrm{C}, 59.37 ; \mathrm{H}, 4.60 ; \mathrm{N}, 7.99$. Found: $\mathrm{C}, 59.06 ; \mathrm{H}, 4.72 ; \mathrm{N}, 7.71$.

Ethyl 2-Diazo-7-methyl-3-oxo-5-(N-tosyl)aminooctanoate (3u). mp 83-84 ${ }^{\circ} \mathrm{C}$; IR (film) 3292, 2957, 2137, 1716, $1649 \mathrm{~cm}^{-1} ;{ }^{1} \mathrm{H}$ NMR $\left(300 \mathrm{MHz}, \mathrm{CDCl}_{3}\right) \delta 0.76(\mathrm{~d}, J=6.6 \mathrm{~Hz}, 3 \mathrm{H}), 0.84(\mathrm{~d}, J=$ $6.6 \mathrm{~Hz}, 3 \mathrm{H}), 1.22-1.31(\mathrm{~m}, 1 \mathrm{H}), 1.32(\mathrm{t}, J=7.2,3 \mathrm{H}), 1.42-1.52(\mathrm{~m}, 1 \mathrm{H}), 1.53-1.64(\mathrm{~m}, 1 \mathrm{H}), 2.41$ $(\mathrm{s}, 3 \mathrm{H}), 2.82(\mathrm{dd}, J=6.0,5.4 \mathrm{~Hz}, 1 \mathrm{H}), 3.01(\mathrm{dd}, J=6.0,16.5 \mathrm{~Hz}, 1 \mathrm{H}), 3.65-3.76(\mathrm{~m}, 1 \mathrm{H}), 4.27(\mathrm{q}$, $J=7.2,2 \mathrm{H}), 5.28(\mathrm{~d}, J=9.0 \mathrm{~Hz}, 1 \mathrm{H}), 7.28(\mathrm{~d}, J=8.1 \mathrm{~Hz}, 2 \mathrm{H}), 7.74(\mathrm{~d}, J=8.1 \mathrm{~Hz}, 2 \mathrm{H}) ;{ }^{13} \mathrm{C}$ NMR $\left(75 \mathrm{MHz}, \mathrm{CDCl}_{3}\right) \delta 14.2,21.3,21.8,22.5,24.4,43.9,44.9,49.3,61.5,127.0,129.4,138.1$, 143.0, 160.9,190.9; EI-MS ( $\mathrm{m} / z$, relative intensity): $395\left(\mathrm{M}^{+}, 4\right), 338$ (6), 310 (4), 240 (24), 155 (65), 91 (100). Anal. Calcd for $\mathrm{C}_{18} \mathrm{H}_{25} \mathrm{~N}_{3} \mathrm{O}_{5} \mathrm{~S}: \mathrm{C}, 54.67 ; \mathrm{H}, 6.37 ; \mathrm{N}, 10.63$. Found: C, 54.66; H, $6.24 ; \mathrm{N}, 10.63$.

Ethyl 2-Diazo-3-oxo-5-( $N$-tosyl)aminoundecanoate (3v). mp $66-67{ }^{\circ} \mathrm{C}$; IR (film) 3293,2929 , 2858, 2137, 1716, $1650 \mathrm{~cm}^{-1}$; ${ }^{1} \mathrm{H}$ NMR $\left(300 \mathrm{MHz}, \mathrm{CDCl}_{3}\right) \delta 0.85(\mathrm{t}, J=7.2 \mathrm{~Hz}, 3 \mathrm{H}), 1.11-1.25$ $(\mathrm{m}, 8 \mathrm{H}), 1.33(\mathrm{t}, J=7.2,3 \mathrm{H}), 1.46-1.52(\mathrm{~m}, 2 \mathrm{H}), 2.41(\mathrm{~s}, 3 \mathrm{H}), 2.82(\mathrm{dd}, J=6.6,4.8 \mathrm{~Hz}, 1 \mathrm{H}), 3.03$ $(\mathrm{dd}, J=6.6,15.9 \mathrm{~Hz}, 1 \mathrm{H}), 3.59-3.66(\mathrm{~m}, 1 \mathrm{H}), 4.27(\mathrm{q}, J=7.2,2 \mathrm{H}), 5.15(\mathrm{~d}, J=8.7 \mathrm{~Hz}, 1 \mathrm{H}), 7.27$ $(\mathrm{d}, J=8.4 \mathrm{~Hz}, 2 \mathrm{H}), 7.72(\mathrm{~d}, J=8.4 \mathrm{~Hz}, 2 \mathrm{H}) ;{ }^{13} \mathrm{C} \mathrm{NMR}\left(75 \mathrm{MHz}, \mathrm{CDCl}_{3}\right) \delta 14.0,14.2,22.3,22.4$, 25.4, 28.7, 31.5, 35.7, 43.7, 51.3, 61.5, 127.0, 129.4, 138.1, 143.0, 161.1, 190.9; EI-MS ( $\mathrm{m} / z$, relative intensity): $423\left(\mathrm{M}^{+}, 3\right), 338$ (23), 268 (31), 310 (11), 155 (100), 91 (98). Anal. Calcd for $\mathrm{C}_{20} \mathrm{H}_{29} \mathrm{~N}_{3} \mathrm{O}_{5} \mathrm{~S}: \mathrm{C}, 56.72 ; \mathrm{H}, 6.90 ; \mathrm{N}, 9.92$. Found: C, 56.75; H, 7.00; N, 9.83.

2-Carboethoxy-3-hydroxy-5-phenylpyrrole (6a). mp 138-140 ${ }^{\circ} \mathrm{C}$; IR (KBr) 3453, 3304, 3278, $1692 \mathrm{~cm}^{-1} ;{ }^{1} \mathrm{H}$ NMR $\left(200 \mathrm{MHz}, \mathrm{CDCl}_{3}\right) \delta 1.37$ (t, $\left.J=7.2 \mathrm{~Hz}, 3 \mathrm{H}\right), 4.37$ (q, $J=7.2 \mathrm{~Hz}, 2 \mathrm{H}$ ), 6.16 $(\mathrm{d}, J=3.2 \mathrm{~Hz}, 1 \mathrm{H}), 7.25-7.52(\mathrm{~m}, 5 \mathrm{H}), 7.91(\mathrm{br}, \mathrm{s}, 1 \mathrm{H}), 8.76(\mathrm{br}, \mathrm{d}, 1 \mathrm{H}) ;{ }^{13} \mathrm{C} \mathrm{NMR}(50 \mathrm{MHz}$, $\left.\mathrm{CDCl}_{3}\right) \delta 14.6,61.2,95.9,106.2,124.9,128.2,128.6,131.1,136.0,155.2,162.0 ; \mathrm{MS} m / z$ (EI) 
$231\left(\mathrm{M}^{+}, 91\right), 203$ (3), 185 (100), 156 (18), 129 (12), 102 (72), 77 (14), 51 (6). Anal. Calcd for $\mathrm{C}_{13} \mathrm{H}_{13} \mathrm{NO}_{3}$ : C, 67.52; H, 5.67; N, 6.06. Found: C, 67.45; H, 5.59; N, 5.91.

2-Carboethoxy-3-hydroxy-5-(2-methylphenyl)pyrrole (6b). mp 91-93 ${ }^{\circ} \mathrm{C}$; IR (KBr) 3489, 3310, 1696, $1677 \mathrm{~cm}^{-1}$; ${ }^{1} \mathrm{H}$ NMR $\left(200 \mathrm{MHz}, \mathrm{CDCl}_{3}\right) \delta 1.37$ (t, $\left.J=7.2 \mathrm{~Hz}, 3 \mathrm{H}\right), 2.43(\mathrm{~s}, 3 \mathrm{H}), 4.35$ (q, $J=7.2 \mathrm{~Hz}, 2 \mathrm{H}), 5.98(\mathrm{~d}, J=2.6 \mathrm{~Hz}, 1 \mathrm{H}), 7.23-7.37(\mathrm{~m}, 4 \mathrm{H}), 7.76(\mathrm{br}, \mathrm{s}, 1 \mathrm{H}), 8.11(\mathrm{br}, \mathrm{s}, 1 \mathrm{H})$; ${ }^{13} \mathrm{C}$ NMR $\left(50 \mathrm{MHz}, \mathrm{CDCl}_{3}\right) \delta 14.5,20.7,60.0,98.8,105.5,126.0,128.3,128.4,130.9,131.5$, 135.7, 135.9, 153.7 (br), 162.0 (br); MS m/z (EI) 245 (M $\left.\mathrm{M}^{+}, 100\right), 222$ (3), 199 (93), 193 (28), 171 (12), 144 (12), 134 (12), 123 (28), 116 (63), 95 (7), 91 (6), 77 (6), 57 (6), 43 (7). Anal. Calcd for $\mathrm{C}_{14} \mathrm{H}_{15} \mathrm{NO}_{3}$ : C, 68.56; H, 6.16; N, 5.71. Found: C, 68.45; H, 6.18; N, 5.61.

2-Carboethoxy-3-hydroxy-5-(3-cyanophenyl)pyrrole (6c). mp $203{ }^{\circ} \mathrm{C}$; IR (KBr) 3501, 3337, 2227, $1669 \mathrm{~cm}^{-1} ;{ }^{1} \mathrm{H}$ NMR $\left(200 \mathrm{MHz}, \mathrm{CDCl}_{3} / \mathrm{d}_{6}\right.$-DMSO) $\delta 1.41(\mathrm{t}, J=7.2 \mathrm{~Hz}, 3 \mathrm{H}), 4.39$ (q, $J=$ $7.2 \mathrm{~Hz}, 2 \mathrm{H}), 6.17(\mathrm{~d}, J=2.8 \mathrm{~Hz}, 1 \mathrm{H}), 7.44-7.56(\mathrm{~m}, 2 \mathrm{H}), 7.89-7.95(\mathrm{~m}, 2 \mathrm{H}), 8.15(\mathrm{~s}, 1 \mathrm{H}), 10.91$ (br, s, $1 \mathrm{H}) ;{ }^{13} \mathrm{C} \mathrm{NMR}\left(50 \mathrm{MHz}, \mathrm{CDCl}_{3} / \mathrm{d}_{6}\right.$-DMSO) $\delta 14.1,59.4,95.7,106.9,112.0,118.1,128.0$, 128.9, 129.9, 132.2, 132.5, 152.7, 161.4; MS m/z (EI) 348 (M+, 4), 284 (6), 256 (14), 241 (2), 210 (20), 178 (5), 171 (43), 155 (57), 127 (14), 107 (22), 91 (100), 65 (39), 57 (31), 39 (18). Anal. Calcd for $\mathrm{C}_{14} \mathrm{H}_{12} \mathrm{~N}_{2} \mathrm{O}_{3}:$ C, 65.62; H, 4.72; N, 10.93. Found: C, 65.81; H, 4.59; N, 10.83.

2-Carboethoxy-3-hydroxy-5-[(E)-cinnamyl]pyrrole (6d). mp $150-152{ }^{\circ} \mathrm{C}$; IR ( $\left.\mathrm{KBr}\right) 3269$, 1680, $1661 \mathrm{~cm}^{-1} ;{ }^{1} \mathrm{H}$ NMR (200 MHz, $\mathrm{CDCl}_{3} / \mathrm{d}_{6}$-DMSO) $\delta 1.37(\mathrm{t}, J=7.2 \mathrm{~Hz}, 3 \mathrm{H}), 4.34$ (q, $J=$ $7.2 \mathrm{~Hz}, 2 \mathrm{H}), 5.94(\mathrm{~d}, J=2.8 \mathrm{~Hz}, 1 \mathrm{H}), 6.88(\mathrm{~d}, J=16.4 \mathrm{~Hz}, 1 \mathrm{H}), 7.06(\mathrm{~d}, J=16.4 \mathrm{~Hz}, 1 \mathrm{H})$, 7.21-7.44 (m, 5H), $7.91(\mathrm{~s}, 1 \mathrm{H}), 11.0(\mathrm{br}, \mathrm{s}, 1 \mathrm{H}) ;{ }^{13} \mathrm{C} \mathrm{NMR}\left(50 \mathrm{MHz}, \mathrm{CDCl}_{3} / \mathrm{d}_{6}-\mathrm{DMSO}\right) \delta 14.0$, $58.9,94.8,105.2,117.6,125.4,126.9,127.9,128.3,133.8,136.2,152.6,161.1 ; \mathrm{MS} \mathrm{m} / \mathrm{z}$ (EI) 257 $\left(\mathrm{M}^{+}, 100\right), 210$ (88), 183 (11), 182 (6), 167 (8), 154 (28), 128 (46), 102 (5), 77 (6), 51 (5), 29 (5). Anal. Calcd for $\mathrm{C}_{15} \mathrm{H}_{15} \mathrm{NO}_{3}$ : C, 70.02; H, 5.88; N, 5.44. Found: C, 70.22; H, 6.08; N, 5.29.

2-Carboethoxy-3-hydroxy-5-(2-furyl)pyrrole (6e). mp 131-133 ${ }^{\circ} \mathrm{C}$; IR (KBr) 3306, 1667, $1564 \mathrm{~cm}^{-1} ;{ }^{1} \mathrm{H}$ NMR $\left(200 \mathrm{MHz}, \mathrm{CDCl}_{3}\right) \delta 1.38(\mathrm{t}, J=7.2 \mathrm{~Hz}, 3 \mathrm{H}), 4.38(\mathrm{q}, J=7.2 \mathrm{~Hz}, 2 \mathrm{H}), 6.06$ $(\mathrm{d}, J=2.6 \mathrm{~Hz}, 1 \mathrm{H}), 6.45-6.47(\mathrm{~m}, 1 \mathrm{H}), 6.56(\mathrm{~d}, J=3.4 \mathrm{~Hz}, 1 \mathrm{H}), 7.41-7.42(\mathrm{~m}, 1 \mathrm{H}), 7.92$ (br, s, 1H), 8.59 (br, s, $1 \mathrm{H}) ;{ }^{13} \mathrm{C}$ NMR $\left(50 \mathrm{MHz}, \mathrm{CDCl}_{3}\right) \delta 14.5,60.1,94.6,105.4,106.2,111.7,126.8$, 142.0, 146.5, 154.4, 162.1; MS m/z (EI) $221\left(\mathrm{M}^{+}, 95\right), 193.3$ (4), 175 (100), 147 (26), 139 (2), 119 (15), 92 (52), 91 (8), 63 (15), 39 (12). Anal. Calcd for $\mathrm{C}_{11} \mathrm{H}_{11} \mathrm{NO}_{4}$ : C, 59.73; H, 5.01; N, 6.33. Found: C, 59.65; H, 4.97; N, 6.13.

2-Benzoyl-3-hydroxy-5-(2-furyl)pyrrole (6f). mp 139-142 ${ }^{\circ} \mathrm{C}$; IR (KBr) 3320, 1590, 1567 $\mathrm{cm}^{-1} ;{ }^{1} \mathrm{H}$ NMR $\left(200 \mathrm{MHz}, \mathrm{CDCl}_{3}\right) \delta 6.14(\mathrm{~d}, J=2.4 \mathrm{~Hz}, 1 \mathrm{H}), 6.50(\mathrm{dd}, J=3.5,1.7 \mathrm{~Hz}, 1 \mathrm{H}), 6.66$ $(\mathrm{d}, J=3.5 \mathrm{~Hz}, 1 \mathrm{H}), 7.44(\mathrm{~d}, J=1.7 \mathrm{~Hz}, 1 \mathrm{H}), 7.50-7.59(\mathrm{~m}, 3 \mathrm{H}), 7.77-7.82(\mathrm{~m}, 2 \mathrm{H}), 8.26(\mathrm{br}, \mathrm{s}$, $1 \mathrm{H}), 10.43$ (br, s, $1 \mathrm{H}) ;{ }^{13} \mathrm{C} \mathrm{NMR}\left(50 \mathrm{MHz}, \mathrm{CDCl}_{3}\right) \delta 94.8,108.2,112.1,116.0,127.5,129.0$, 129.8, 131.6, 137.7, 142.7, 145.9, 159.3, 184.0; MS m/z (EI) 253 ( $\left.\mathrm{M}^{+}, 100\right), 236$ (5), 224 (4), 196 
(4), 176 (30), 147 (7), 120 (9), 105 (37), 92 (24), 91 (3), 77 (49), 65 (20), 51 (17), 39 (15). Anal. Calcd for $\mathrm{C}_{15} \mathrm{H}_{11} \mathrm{NO}_{3}:$ C, $71.14 ; \mathrm{H}, 4.38 ; \mathrm{N}, 5.53$. Found: $\mathrm{C}, 70.98 ; \mathrm{H}, 4.37 ; \mathrm{N}, 5.46$.

2-Benzoyl-3-hydroxy-5-[(E)-cinnamyl]pyrrole (6g). mp 159-161 ${ }^{\circ} \mathrm{C}$; IR (KBr) 3322, 2261, 1626, 1592, 1550, $1503 \mathrm{~cm}^{-1} ;{ }^{1} \mathrm{H}$ NMR $\left(200 \mathrm{MHz}, \mathrm{CDCl}_{3}\right) \delta 6.10(\mathrm{~d}, J=2 \mathrm{~Hz}, 1 \mathrm{H}), 6.79(\mathrm{~d}, J=$ $16.5 \mathrm{~Hz}, 1 \mathrm{H}), 7.00(\mathrm{~d}, J=16.5 \mathrm{~Hz}, 1 \mathrm{H}), 7.28-7.54(\mathrm{~m}, 8 \mathrm{H}), 7.68-7.72(\mathrm{~m}, 2 \mathrm{H}), 8.41(\mathrm{br}, \mathrm{s}, 1 \mathrm{H})$, 10.40 (br, s, $1 \mathrm{H}) ;{ }^{13} \mathrm{C}$ NMR $\left(50 \mathrm{MHz}, \mathrm{CDCl}_{3}\right) \delta 96.3,116.6,117.3,126.6,127.5,128.5,128.8$, 128.9, 131.6, 132.0, 135.9, 137.7, 138.0, 159.5, 183.8; MS m/z (EI) 289 (M $\left.\mathrm{M}^{+}, 100\right), 288$ (27), 270 (10), 212 (13), 156 (6), 128 (23), 105 (49), 77 (31), 51 (7). Anal. Calcd for $\mathrm{C}_{19} \mathrm{H}_{15} \mathrm{NO}_{2}$ : C, 78.87; H, 5.23; N, 4.84. Found: C, 78.80; H, 5.21; N, 4.74.

2-Acetyl-3-hydroxy-5-(4-methoxyphenyl)pyrrole (6h). mp 178-180 ${ }^{\circ} \mathrm{C}$ (decomposed); IR (KBr) 3272, 1614, 1585, $1534 \mathrm{~cm}^{-1}$; ${ }^{1} \mathrm{H}$ NMR $\left(200 \mathrm{MHz}, \mathrm{CDCl}_{3}\right) \delta 2.48(\mathrm{~s}, 3 \mathrm{H}), 3.85(\mathrm{~s}, 3 \mathrm{H})$, $6.01(\mathrm{~d}, J=2.8 \mathrm{~Hz}, 1 \mathrm{H}), 6.93(\mathrm{~d}, J=8.6 \mathrm{~Hz}, 2 \mathrm{H}), 7.62(\mathrm{~d}, J=8.6 \mathrm{~Hz}, 2 \mathrm{H}), 9.47$ (br, s, 1H), 10.59 (br, s, 1H); MS m/z (EI) 231 (M $\left.\mathrm{M}^{+}, 100\right), 216$ (93), 202 (9), 188 (5), 174 (8), 161 (15), 146 (4), 133 (18), 118 (7), 117 (8), 102 (3), 89 (12), 77 (4), 63 (6), 43 (13). Anal. Calcd for $\mathrm{C}_{13} \mathrm{H}_{13} \mathrm{NO}_{3}$ : C, 67.52; H, 5.67; N, 6.06. Found: C, 67.41; H, 5.71; N, 6.01.

2-Carboethoxy-3-hydroxy-4-methyl-5-phenylpyrrole (6i). mp 94-95 ${ }^{\circ} \mathrm{C}$; IR (film) 3306, 2980, $1676 \mathrm{~cm}^{-1} ;{ }^{1} \mathrm{H}$ NMR $\left(300 \mathrm{MHz}, \mathrm{CDCl}_{3}\right) \delta 1.36(\mathrm{t}, J=7.2 \mathrm{~Hz}, 3 \mathrm{H}), 2.14(\mathrm{~s}, 3 \mathrm{H}), 4.35$ (q, $J$ $=7.2 \mathrm{~Hz}, 2 \mathrm{H}), 7.26-7.51(\mathrm{~m}, 5 \mathrm{H}) ;{ }^{13} \mathrm{C} \mathrm{NMR}\left(75 \mathrm{MHz}, \mathrm{CDCl}_{3}\right) \delta$ 7.9, 14.6, 60.0, 105.2, 127.0, 127.9, 128.8, 132.2; EI-MS (m/z, relative intensity): $245\left(\mathrm{M}^{+}, 76\right), 199$ (100), 171 (26), $116(81)$. Anal. Calcd for $\mathrm{C}_{14} \mathrm{H}_{15} \mathrm{NO}_{3}$ : C, 68.56; H, 6.16; N, 5.71. Found: C, 68.47; H, 6.30; N, 5.55.

2-Carboethoxy-3-hydroxy-4-methyl-5-(4-methoxyphenyl)pyrrole (6j). mp 77-78 ${ }^{\circ} \mathrm{C}$; IR (film) 3313, 2980, $1672 \mathrm{~cm}^{-1}$; ${ }^{1} \mathrm{H}$ NMR $\left(300 \mathrm{MHz}, \mathrm{CDCl}_{3}\right) \delta 8.47(\mathrm{br}, \mathrm{s}, 1 \mathrm{H}), 7.88(\mathrm{br}, \mathrm{d}, J=$ 18.3), 7.42 (d, $J=9.0 \mathrm{~Hz}, 2 \mathrm{H}), 6.97(\mathrm{~d}, J=9.0 \mathrm{~Hz}, 2 \mathrm{H}), 4.34$ (q, $J=7.2 \mathrm{~Hz}, 2 \mathrm{H}), 3.84(\mathrm{~s}, 3 \mathrm{H})$, $2.11(\mathrm{~s}, 3 \mathrm{H}), 1.36(\mathrm{t}, J=7.2 \mathrm{~Hz}, 3 \mathrm{H}) ;{ }^{13} \mathrm{C} \mathrm{NMR}\left(75 \mathrm{MHz}, \mathrm{CDCl}_{3}\right) \delta 159.3,128.3,124.8,114.2$, 104.5, 59.90, 55.30, 14.62, 7.84; EI-MS ( $m / z$, relative intensity): 275 ( $\left.\mathrm{M}^{+}, 88.8\right), 229$ (100), 199 (35.5), 146 (66.4); Anal. Calcd for $\mathrm{C}_{15} \mathrm{H}_{17} \mathrm{NO}_{4}$ : C, 65.44; H, 6.22; N, 5.09. Found: C, 65.43; H, $6.18 ; \mathrm{N}, 4.79$.

2-Carboethoxy-3-hydroxy-5-(2-methylphenyl)-4-phenylpyrrole (6k). mp $179-180{ }^{\circ} \mathrm{C}$; IR (film) 3288, 2981, 1677, 1655, $1303 \mathrm{~cm}^{-1}$; ${ }^{1} \mathrm{H} \mathrm{NMR}\left(300 \mathrm{MHz}, \mathrm{CDCl}_{3}\right) \delta 1.36(\mathrm{t}, J=7.2 \mathrm{~Hz}, 3 \mathrm{H})$, 1.99 (s, 3H), 4.38 (q, $J=7.2 \mathrm{~Hz}, 2 \mathrm{H}), 7.10-7.34(\mathrm{~m}, 9 \mathrm{H}), 8.13(\mathrm{br}, \mathrm{d}, 1 \mathrm{H}), 8.66(\mathrm{br}, \mathrm{s}, 1 \mathrm{H}) ;{ }^{13} \mathrm{C}$ NMR $\left(75 \mathrm{MHz}, \mathrm{CDCl}_{3}\right) \delta 14.6,19.8,60.2,111.8,125.9,126.0,128.0,128.2,129.0,130.2,130.5$, 132.0, 132.8, 137.5; EI-MS (m/z, relative intensity): $321\left(\mathrm{M}^{+}, 72\right), 275$ (100), 260 (30), 191 (33); Anal. Calcd for $\mathrm{C}_{20} \mathrm{H}_{19} \mathrm{NO}_{3}: \mathrm{C}, 74.75 ; \mathrm{H}, 5.96 ; \mathrm{N}, 4.36$. Found: $\mathrm{C}, 74.83 ; \mathrm{H}, 5.91 ; \mathrm{N}, 4.13$.

2-Carboethoxy-5-(4-chlorophenyl)-3-hydroxy-4-phenylpyrrole (61). mp 136-137 ${ }^{\circ} \mathrm{C}$; IR (film) 3292, 2982, $1678 \mathrm{~cm}^{-1}$; ${ }^{1} \mathrm{H}$ NMR $\left(300 \mathrm{MHz}, \mathrm{CDCl}_{3}\right) \delta 1.39$ (t, $\left.J=7.2 \mathrm{~Hz}, 3 \mathrm{H}\right), 4.39$ (q, $J=$ 
$7.2 \mathrm{~Hz}, 2 \mathrm{H}), 7.22-7.34(\mathrm{~m}, 9 \mathrm{H}), 8.10(\mathrm{br}, \mathrm{s}, 2 \mathrm{H}) ;{ }^{13} \mathrm{C} \mathrm{NMR}\left(75 \mathrm{MHz}, \mathrm{CDCl}_{3}\right) \delta 14.6,34.2,60.4$, 126.6, 128.4, 129.0, 129.5, 130.2, 131.8; EI-MS ( $\mathrm{m} / z$, relative intensity): $341\left(\mathrm{M}^{+}, 82\right), 295(100)$, 260 (20), 212 (36), 176 (18). Anal. Calcd for $\mathrm{C}_{20} \mathrm{H}_{19} \mathrm{NO}_{3}$ : C, 66.77; H, 4.72; N, 4.10. Found: C, $66.59 ; \mathrm{H}, 4.80 ; \mathrm{N}, 3.83$.

2-Carboethoxy-3-hydroxy-5-isobutylpyrrole (6m). mp 95-96 ${ }^{\circ} \mathrm{C}$; IR (film) 3289, 2951, 1676, $1659 \mathrm{~cm}^{-1} ;{ }^{1} \mathrm{H}$ NMR $\left(300 \mathrm{MHz}, \mathrm{CDCl}_{3}\right) \delta 0.92(\mathrm{~d}, J=7.2 \mathrm{~Hz}, 6 \mathrm{H}), 1.36$ (br, s, 3H), $1.88(\mathrm{~m}, 1 \mathrm{H})$, $2.39(\mathrm{~d}, J=7.2 \mathrm{~Hz}, 2 \mathrm{H}), 4.34(\mathrm{br}, \mathrm{s}, 2 \mathrm{H}), 5.62(\mathrm{~d}, J=2.4,1 \mathrm{H}), 6.58(\mathrm{br}, 0.5 \mathrm{H}), 7.92(\mathrm{br}, 1 \mathrm{H})$, $8.93(\mathrm{br}, 0.5 \mathrm{H}) ;{ }^{13} \mathrm{C} \mathrm{NMR}\left(75 \mathrm{MHz}, \mathrm{CDCl}_{3}\right) \delta 14.6,22.3,28.8,37.7,59.7,97.0,103.7,137.2$, 155.2, 162.4; EI-MS (m/z, relative intensity): $211\left(\mathrm{M}^{+}, 48\right), 168$ (100), 140 (41), 122 (67), 28 (43). Anal. Calcd for $\mathrm{C}_{11} \mathrm{H}_{17} \mathrm{NO}_{3}$ : C, 62.54; H, 8.11; N, 6.63. Found: C, 62.53; H, 8.06; N, 6.35.

2-Carboethoxy-5-hexyl-3-hydroxypyrrole (6n). $\mathrm{mp}$ 69-70 ${ }^{\circ} \mathrm{C}$; IR (film) 3448, 3301, 2955, 2922, 2856, $1677 \mathrm{~cm}^{-1} ;{ }^{1} \mathrm{H}$ NMR $\left(300 \mathrm{MHz}, \mathrm{CDCl}_{3}\right) \delta 0.88(\mathrm{t}, J=6.6 \mathrm{~Hz}, 3 \mathrm{H}), 1.29-1.37(\mathrm{~m}, 9 \mathrm{H})$, $1.55 \sim 1.65(\mathrm{~m}, 2 \mathrm{H}), 2.52(\mathrm{t}, J=7.2 \mathrm{~Hz}, 2 \mathrm{H}), 4.33(\mathrm{br}, \mathrm{s}, 2 \mathrm{H}), 5.62(\mathrm{~d}, J=2.7,1 \mathrm{H}), 6.57$ (br, $0.5 \mathrm{H})$, $7.90(\mathrm{br}, 1 \mathrm{H}), 8.74(\mathrm{br}, 0.5 \mathrm{H}) ;{ }^{13} \mathrm{C}$ NMR $\left(75 \mathrm{MHz}, \mathrm{CDCl}_{3}\right) \delta 13.9,14.5,155.3,22.5,28.3,28.8$, 28.9, 31.4, 59.7, 96.2; EI-MS ( $\mathrm{m} / \mathrm{z}$, relative intensity): 239 ( $\left.\mathrm{M}^{+}, 34\right), 194$ (14), 168 (34), 122 (58), 67 (58) 41 (64), 29 (100). Anal. Calcd for $\mathrm{C}_{13} \mathrm{H}_{21} \mathrm{NO}_{3}$ : C, 65.25; H, 8.84; N, 5.85. Found: C, $65.38 ; \mathrm{H}, 8.57 ; \mathrm{N}, 5.68$.

\section{$S$-p-tolyl-p-toluenesulfonothioate (11). ${ }^{1}$}

M.p. 88-90 ${ }^{\circ} \mathrm{C}$; IR (KBr) 1590, 1487, 1323, 1140, 804, 652, $585 \mathrm{~cm}^{-1} ;{ }^{1} \mathrm{H}$ NMR $(200 \mathrm{MHz}$, $\left.\mathrm{CDCl}_{3}\right) \delta 2.83(\mathrm{~s}, 3 \mathrm{H}), 2.42(\mathrm{~s}, 3 \mathrm{H}), 7.12-7.23(\mathrm{~m}, 6 \mathrm{H}), 7.46(\mathrm{~d}, J=8.8 \mathrm{~Hz}, 2 \mathrm{H}) ;{ }^{13} \mathrm{C}$ NMR $(50$ $\left.\mathrm{MHz}, \mathrm{CDCl}_{3}\right) \delta 144.6,142.0,140.4,136.5,130.2,129.3,127.6,124.5 .21 .63 .21 .46$; MS (EI) $\left(\mathrm{m} / \mathrm{z}\right.$, relative intensity) $278\left(\mathrm{M}^{+}, 52\right), 155$ (32), 139 (100), 123 (44), 91 (82), 65 (23), 45 (19).

3-Carboethoxy-5-phenyl- $N$-tosyl-2-pyrrolidinone (7a). Amorphous solid; the major isomer: IR (film) 2976, 1728, 1367, $1170 \mathrm{~cm}^{-1}$; ${ }^{1} \mathrm{H}$ NMR (300 MHz, $\left.\mathrm{CDCl}_{3}\right) \delta 1.22-1.29(\mathrm{~m}, 3 \mathrm{H})$, 2.15-2.22 (m, 1H), $2.40(\mathrm{~s}, 3 \mathrm{H}), 2.93-3.04(\mathrm{~m}, 1 \mathrm{H}), 3.70-3.76(\mathrm{~m}, 1 \mathrm{H}), 4.13-4.25(\mathrm{~m}, 2 \mathrm{H})$, 5.49-5.52 (m, 1H), 7.11-7.32 (m, 7H), 7.50-7.62 (m, 2H); ${ }^{13} \mathrm{C} \mathrm{NMR}\left(75 \mathrm{MHz}, \mathrm{CDCl}_{3}\right) \delta 13.9$, 21.6, 32.3, 47.8, 61.2, 62.1, 125.9, 127.0, 128.3, 128.5, 128.9, 134.8, 139.8, 145.2, 167.6, 168.5; EI-MS ( $/ \mathrm{z}$, relative intensity): $388\left[(\mathrm{M}+1)^{+}, 6\right], 323(89), 250(31), 232(38), 186(47), 155(25)$, 91 (100). Anal. Calcd for $\mathrm{C}_{20} \mathrm{H}_{21} \mathrm{NO}_{5} \mathrm{~S}$ : C, 62.00; H, 5.46; N, 3.62. Found: C, 62.11; H, 5.57; N, 3.44 .

3-Carboethoxy-5-(2-methylphenyl)- $N$-tosyl-2-pyrrolidinone (7b). Amorphous solid; the major isomer: IR (film) 2981, 1747, 1729, $1170 \mathrm{~cm}^{-1} ;{ }^{1} \mathrm{H} \mathrm{NMR}\left(300 \mathrm{MHz}, \mathrm{CDCl}_{3}\right) \delta 1.27(\mathrm{t}, J=$ $7.2 \mathrm{~Hz}, 3 \mathrm{H}), 2.42(\mathrm{~s}, 6 \mathrm{H}), 2.04-2.12(\mathrm{~m}, 1 \mathrm{H}), 2.92-3.03(\mathrm{~m}, 1 \mathrm{H}), 3.65-3.72(\mathrm{~m}, 1 \mathrm{H}), 4.21(\mathrm{q}, J=$ $7.2 \mathrm{~Hz}, 2 \mathrm{H}), 5.74-5.77(\mathrm{~m}, 1 \mathrm{H}), 6.78(\mathrm{~d}, J=7.8 \mathrm{~Hz}, 1 \mathrm{H}), 6.98-7.04(\mathrm{~m}, 1 \mathrm{H}), 7.20-7.27(\mathrm{~m}, 4 \mathrm{H})$, $7.69(\mathrm{~d}, J=8.1 \mathrm{~Hz}, 2 \mathrm{H}) ;{ }^{13} \mathrm{C} \mathrm{NMR}\left(75 \mathrm{MHz}, \mathrm{CDCl}_{3}\right) \delta 14.0,19.1,21.6,31.3,47.5,57.7,62.1$, 
124.2, 126.2, 127.9, 128.8, 129.2, 131.2, 134.5,134.7, 137.7, 145.3, 167.6, 168.7. EI-MS (m/z, relative intensity): $401\left(\mathrm{M}^{+}, 26\right), 337$ (28), 264 (45), 246 (45), 200 (47), 91 (100). Anal. Calcd for $\mathrm{C}_{21} \mathrm{H}_{23} \mathrm{NO}_{5} \mathrm{~S}: \mathrm{C}, 62.82 ; \mathrm{H}, 5.77 ; \mathrm{N}, 3.49$. Found: $\mathrm{C}, 62.54 ; \mathrm{H}, 5.74 ; \mathrm{N}, 3.25$.

3-Carboethoxy-5-(4-chlorophenyl)- $N$-tosyl-2-pyrrolidinone (7c). Amorphous solid; the major isomer: IR (film) 1729, 1729, $1170 \mathrm{~cm}^{-1} ;{ }^{1} \mathrm{H}$ NMR $\left(300 \mathrm{MHz}, \mathrm{CDCl}_{3}\right) \delta 1.23-1.28(\mathrm{~m}, 3 \mathrm{H})$, 2.10-2.18 (m, 1H), $2.42(\mathrm{~s}, 3 \mathrm{H}), 2.92-3.02(\mathrm{~m}, 1 \mathrm{H}), 3.61-3.72(\mathrm{~m}, 1 \mathrm{H}), 4.13-4.24(\mathrm{~m}, 2 \mathrm{H})$, 5.45-5.49 (m, 1H), $7.10(\mathrm{~d}, J=8.4 \mathrm{~Hz}, 2 \mathrm{H}), 7.65(\mathrm{~d}, J=8.4 \mathrm{~Hz}, 2 \mathrm{H}), 7.71-8.31(\mathrm{~m}, 4 \mathrm{H}) ;{ }^{13} \mathrm{C}$ NMR $\left(75 \mathrm{MHz}, \mathrm{CDCl}_{3}\right) \delta 13.9,21.6,32.2,47.7,60.6,62.2,127.3,128.4,128.7,129.0,134.1$, 134.7, 138.5, 145.5, 167.4,168.3; EI-MS ( $/ z / z$, relative intensity): $422\left[(\mathrm{M}+1)^{+}, 5\right], 357$ (58), 266 (40), 220 (42), 155(29), 91 (100). Anal. Calcd for $\mathrm{C}_{20} \mathrm{H}_{20} \mathrm{ClNO}_{5} \mathrm{~S}$ : C, 56.94; H, 4.78; N, 3.32. Found: C, 56.85; H, 4.87; N, 3.22.

3-Carboethoxy-5-(3-cyanophenyl)- $\mathrm{N}$-tosyl-2-pyrrolidinone (7d). Amorphous solid; the major isomer: IR (film) 2231, 1729, 1560, $1170 \mathrm{~cm}^{-1} ;{ }^{1} \mathrm{H} \mathrm{NMR}\left(300 \mathrm{MHz}, \mathrm{CDCl}_{3}\right) \delta 1.27(\mathrm{t}, J=$ 7.2, 3H), 2.10-2.18 (m, 1H), $2.45(\mathrm{~s}, 3 \mathrm{H}), 2.95-3.06(\mathrm{~m}, 1 \mathrm{H}), 3.64-3.70(\mathrm{~m}, 1 \mathrm{H}), 4.15-4.26(\mathrm{~m}$, 2H), 5.50-5.53 (m, 1H), 7.20-7.68 (m, 8H); $\left.{ }^{13} \mathrm{C} \mathrm{NMR} \mathrm{(75} \mathrm{MHz,} \mathrm{CDCl}_{3}\right) \delta 13.9,21.7,32.1,47.6$, $60.5,62.4,113.1,117.9,128.4,129.4,129.9,130.5,131.4,131.9,134.5,141.6,146.0$, 167.2,168.1; EI-MS ( $\mathrm{m} / \mathrm{z}$, relative intensity): $413\left[(\mathrm{M}+1)^{+}, 3\right], 258(100), 212(53), 184(45), 105$ (29), 91 (51); Anal. Calcd for $\mathrm{C}_{21} \mathrm{H}_{20} \mathrm{~N}_{2} \mathrm{O}_{5} \mathrm{~S}: \mathrm{C}, 61.15 ; \mathrm{H}, 4.89$; N, 6.79. Found: C, 61.07; H, 5.03; $\mathrm{N}, 6.65$.

3-Carboethoxy-5-furyl- $N$-tosyl-2-pyrrolidinone (7e). Amorphous solid; the major isomer: IR (film) 1730, $1171 \mathrm{~cm}^{-1}$; ${ }^{1} \mathrm{H}$ NMR $\left(300 \mathrm{MHz}, \mathrm{CDCl}_{3}\right) \delta 1.27-1.32(\mathrm{~m}, 3 \mathrm{H}), 2.31-2.40(\mathrm{~m}, 1 \mathrm{H})$, $2.38(\mathrm{~s}, 3 \mathrm{H}), 2.89-3.00(\mathrm{~m}, 1 \mathrm{H}), 3.91-3.98(\mathrm{~m}, 1 \mathrm{H}), 4.20-4.28(\mathrm{~m}, 2 \mathrm{H}), 5.52(\mathrm{~d}, J=8.4 \mathrm{~Hz}, 1 \mathrm{H})$, 6.37-6.49 (m, 2H), $7.17(\mathrm{~d}, J=8.4 \mathrm{~Hz}, 2 \mathrm{H}), 7.47(\mathrm{~d}, J=8.4 \mathrm{~Hz}, 2 \mathrm{H}) ;{ }^{13} \mathrm{C}$ NMR $\left(75 \mathrm{MHz}, \mathrm{CDCl}_{3}\right)$ $\delta 14.0,21.6,29.0,48.5,53.8,62.1,110.0,110.4,128.3,129.2,134.4,142.6,145.0,151.5$, 167.5,168.0; EI-MS ( $\mathrm{m} / \mathrm{z}$, relative intensity): $377\left(\mathrm{M}^{+}, 2\right), 222$ (100), 176 (96), 148 (28), 91 (47). Anal. Calcd for $\mathrm{C}_{18} \mathrm{H}_{19} \mathrm{NO}_{6} \mathrm{~S}: \mathrm{C}, 57.28 ; \mathrm{H}, 5.07 ; \mathrm{N}, 3.71$. Found: C, 57.28; H, 5.10; N, 3.58.

3-Carboethoxy-4-methyl-5-phenyl- $\boldsymbol{N}$-tosyl-2-pyrrolidinone (7h). Amorphous solid; the major isomer: IR (film) 2979, 1748, 1730, $1172 \mathrm{~cm}^{-1} ;{ }^{1} \mathrm{H} \mathrm{NMR}\left(300 \mathrm{MHz}, \mathrm{CDCl}_{3}\right) \delta 1.21(\mathrm{t}, J=$ $7.2 \mathrm{~Hz}, 3 \mathrm{H}), 2.00(\mathrm{~s}, 3 \mathrm{H}), 2.43(\mathrm{~s}, 3 \mathrm{H}), 3.79(\mathrm{~m}, 1 \mathrm{H}), 3.90(\mathrm{~d}, J=9.9 \mathrm{~Hz}, 1 \mathrm{H}), 4.08-4.23(\mathrm{~m}, 2 \mathrm{H})$, $5.53(\mathrm{~d}, J=6.9 \mathrm{~Hz}, 1 \mathrm{H}), 5.74-5.77(\mathrm{~m}, 1 \mathrm{H}), 7.04-7.31(\mathrm{~m}, 11 \mathrm{H}), 7.56(\mathrm{~d}, J=8.1 \mathrm{~Hz}, 2 \mathrm{H}) ;{ }^{13} \mathrm{C}$ NMR $\left(75 \mathrm{MHz}, \mathrm{CDCl}_{3}\right) \delta 14.0,16.8,21.6,40.8,56.1,62.0,68.9,126.3,127.3,128.3,128.4$, $128.5,128.6,128.7,129.1,129.2,135.3,138.8,145.0,167.6,168.6$; EI-MS $(\mathrm{m} / \mathrm{z}$, relative intensity): $402\left[(\mathrm{M}+1)^{+}, 3\right], 337$ (91), 264 (35), 200 (27), 155 (33), 132 (34), 91 (100); Anal. Calcd for $\mathrm{C}_{21} \mathrm{H}_{23} \mathrm{NO}_{6} \mathrm{~S}$ : C, 62.84; H, 5.74; N, 3.49. Found: C, 63.24; H, 6.04; N, 3.34; HRMS calcd for $\mathrm{C}_{21} \mathrm{H}_{23} \mathrm{NO}_{5} \mathrm{~S}: 401.1296$, found: 401.1308 . 
3-Carboethoxy-4-methyl-5-(4-methoxyphenyl)- $\mathrm{N}$-tosyl-2-pyrrolidinone (7i). Amorphous solid; the major isomer: IR (film) 2922, 1750, 1731, $1204 \mathrm{~cm}^{-1} ;{ }^{1} \mathrm{H}$ NMR $\left(300 \mathrm{MHz}, \mathrm{CDCl}_{3}\right) \delta$ $1.21(\mathrm{t}, J=7.2 \mathrm{~Hz}, 3 \mathrm{H}), 2.00(\mathrm{~s}, 3 \mathrm{H}), 2.43(\mathrm{~s}, 3 \mathrm{H}), 3.79(\mathrm{~m}, 1 \mathrm{H}), 3.90(\mathrm{~d}, J=9.9 \mathrm{~Hz}, 1 \mathrm{H})$, 4.08-4.23 (m, 2H), 5.74-5.77 (m, 1H), $5.53(\mathrm{~d}, J=6.9 \mathrm{~Hz}, 1 \mathrm{H}), 7.04-7.31(\mathrm{~m}, 11 \mathrm{H}), 7.56(\mathrm{~d}, J=$ $8.1 \mathrm{~Hz}, 2 \mathrm{H}) ;{ }^{13} \mathrm{C} \mathrm{NMR}\left(75 \mathrm{MHz}, \mathrm{CDCl}_{3}\right) \delta 14.0,16.6,21.6,40.8,55.2,56.1,61.9,68.6,113.7$, $114.0,127.7,128.2,128.5,128.7,129.1,129.2$, 130.6, 135.4, 144.9, 159.5, 167.7, 168.5; EI-MS ( $\mathrm{m} / \mathrm{z}$, relative intensity): $431\left(\mathrm{M}^{+}, 44\right), 276$ (100), 230 (90), 202 (34), 162 (47), 134 (35), 91 (71). HRMS calcd for $\mathrm{C}_{22} \mathrm{H}_{25} \mathrm{NO}_{6} \mathrm{~S}: 431.1402$, found: 431.1394 .

3-Carboethoxy-5-(2-methylphenyl)-4-phenyl- $N$-tosyl-2-pyrrolidinone (7j). The major isomer: M.p. $179-180{ }^{\circ} \mathrm{C}$; IR (film) 2982, $1730,1170 \mathrm{~cm}^{-1} ;{ }^{1} \mathrm{H}$ NMR $\left(300 \mathrm{MHz}, \mathrm{CDCl}_{3}\right) \delta 1.21(\mathrm{t}$, $J=7.2 \mathrm{~Hz}, 3 \mathrm{H}), 2.00(\mathrm{~s}, 3 \mathrm{H}), 2.43(\mathrm{~s}, 3 \mathrm{H}), 3.79(\mathrm{~m}, 1 \mathrm{H}), 3.90(\mathrm{~d}, J=9.9 \mathrm{~Hz}, 1 \mathrm{H}), 4.08-4.23(\mathrm{~m}$, $2 \mathrm{H}), 5.74-5.77(\mathrm{~m}, 1 \mathrm{H}), 5.53(\mathrm{~d}, J=6.9 \mathrm{~Hz}, 1 \mathrm{H}), 7.04-7.31(\mathrm{~m}, 11 \mathrm{H}), 7.56(\mathrm{~d}, J=8.1 \mathrm{~Hz}, 2 \mathrm{H})$; ${ }^{13} \mathrm{C}$ NMR $\left(75 \mathrm{MHz}, \mathrm{CDCl}_{3}\right) \delta 13.9,19.1,21.7,51.2,56.0,62.2,64.4,126.0,126.3,127.2,168.3$, 127.9, 128.1, 128.6, 129.1, 129.2, 130.4, 135.2, 136.2, 137.2, 137.8, 145.2, 167.3; EI-MS (m/z, relative intensity): $477\left(\mathrm{M}^{+}, 7\right), 404$ (16), 322 (33), 274 (36), 176 (100), 146 (70), 131 (80), 118 (55), 91 (85); Anal. Calcd for $\mathrm{C}_{27} \mathrm{H}_{27} \mathrm{NO}_{5} \mathrm{~S}$ : C, 67.90; H, 5.70; N, 2.93. Found: C, 67.77; H, 5.87; $\mathrm{N}, 2.70$.

One of the minor isomers: ${ }^{1} \mathrm{H}$ NMR $\left(300 \mathrm{MHz}, \mathrm{CDCl}_{3}\right) \delta 1.19(\mathrm{t}, J=7.2 \mathrm{~Hz}, 3 \mathrm{H}), 1.77(\mathrm{~s}, 3 \mathrm{H})$, $2.42(\mathrm{~s}, 3 \mathrm{H}), 4.04(\mathrm{~d}, J=13.5 \mathrm{~Hz}, 1 \mathrm{H}), 4.05-4.21(\mathrm{~m}, 2 \mathrm{H}), 4.38-4.51(\mathrm{~m}, 1 \mathrm{H}), 5.97(\mathrm{~d}, J=8.1 \mathrm{~Hz}$, $1 \mathrm{H}), 6.66-6.73(\mathrm{~m}, 3 \mathrm{H}), 6.89-7.27(\mathrm{~m}, 8 \mathrm{H}), 7.63(\mathrm{~d}, J=8.4 \mathrm{~Hz}, 2 \mathrm{H}) ;{ }^{13} \mathrm{C} \mathrm{NMR}\left(75 \mathrm{MHz}, \mathrm{CDCl}_{3}\right)$ $\delta 13.9,19.0,21.7,48.5,53.1,61.1,62.1,125.4,125.6,127.9,128.0,128.2,128.6,129.0,129.2$, $130.8,133.1,133.5,134.6,137.0,145.4,166.7,168.0$.

3-Carboethoxy-5-(4-chlorophenyl)-4-phenyl- $\mathrm{N}$-tosyl-2-pyrrolidinone (7k). Amorphous solid; the major isomer: IR (film) 2982, $1730,1170 \mathrm{~cm}^{-1} ;{ }^{1} \mathrm{H}$ NMR $\left(300 \mathrm{MHz}, \mathrm{CDCl}_{3}\right) \delta 1.20(\mathrm{t}, J$ $=7.2 \mathrm{~Hz}, 3 \mathrm{H}), 2.44(\mathrm{~s}, 3 \mathrm{H}), 4.03(\mathrm{~d}, J=13.2 \mathrm{~Hz}, 1 \mathrm{H}), 4.08-4.22(\mathrm{~m}, 2 \mathrm{H}), 4.44-5.51(\mathrm{~m}, 1 \mathrm{H}), 5.62$ $(\mathrm{d}, J=7.8 \mathrm{~Hz}, 1 \mathrm{H}), 6.59(\mathrm{~d}, J=8.4 \mathrm{~Hz}, 2 \mathrm{H}), 6.76-6.80(\mathrm{~m}, 2 \mathrm{H}), 7.01-7.31(\mathrm{~m}, 7 \mathrm{H}), 7.72(\mathrm{~d}, J=$ $8.4 \mathrm{~Hz}, 2 \mathrm{H}) ;{ }^{13} \mathrm{C}$ NMR $\left(75 \mathrm{MHz}, \mathrm{CDCl}_{3}\right) \delta 13.9,21,6,47.9,51.3,62.3,64.9,126.8,127.2,127.8$, $127.9,128.2$, 128.3, 128.4, 128.5, 128.7, 129.2, 129.4, 133.3, 133.5, 134.1, 134.2, 134.6, 137.3, 145.6, 166.6, 167.7; EI-MS ( $m / z$, relative intensity): $498\left[(\mathrm{M}+1)^{+}, 5\right], 433(8), 342$ (31), 294 (22), 176 (100), 166 (31), 131 (62), 91 (45). HRMS calcd for $\mathrm{C}_{26} \mathrm{H}_{24} \mathrm{ClNO}_{5} \mathrm{~S}$ : 497.1063, found: 497.1063.

3-Carboethoxy-5-isobutyl- $\boldsymbol{N}$-tosyl-2-pyrrolidinone (7l). Amorphous solid; the major isomer: IR (film) 2960, 1748, 1729, $1170 \mathrm{~cm}^{-1} ;{ }^{1} \mathrm{H}$ NMR (300 MHz, $\left.\mathrm{CDCl}_{3}\right) \delta$ 0.97-1.03 (m, 6H), 1.21-1.27 (m, 3H), 1.40-1.96 (m, 3H), $2.44(\mathrm{~s}, 3 \mathrm{H}), 2.21-2.72(\mathrm{~m}, 2 \mathrm{H}), 3.54-3.60(\mathrm{~m}, 1 \mathrm{H})$, 4.13-4.22 (m, 2H), 4.34-4.56 (m, 1H), $7.34(\mathrm{~d}, J=8.4 \mathrm{~Hz}, 2 \mathrm{H}), 7.94(\mathrm{~d}, J=8.4 \mathrm{~Hz}, 2 \mathrm{H}) ;{ }^{13} \mathrm{C}$ NMR $\left(75 \mathrm{MHz}, \mathrm{CDCl}_{3}\right) \delta 13.9,21.2,21.6,23.7,25.4,27.9,44.2,47.7,57.2,61.9,128.4,129.5$, 135.5, 145.2, 167.8, 168.3; EI-MS (m/z, relative intensity): $\left.368[\mathrm{M}+1]^{+}, 6\right], 303(20), 230(26)$, 
155 (80), 91 (100). Anal. Calcd for $\mathrm{C}_{18} \mathrm{H}_{25} \mathrm{NO}_{5} \mathrm{~S}: \mathrm{C}, 58.83 ; \mathrm{H}, 6.86 ; \mathrm{N}, 3.81$. Found: C, 59.11; H, $6.89 ; \mathrm{N}, 3.66$.

3-Carboethoxy-5-hexyl- $\boldsymbol{N}$-tosyl-2-pyrrolidinone (7m). Amorphous solid; the major isomer: IR (film) 2929, 2859, 1729, $1169 \mathrm{~cm}^{-1}$; ${ }^{1} \mathrm{H}$ NMR $\left(300 \mathrm{MHz}, \mathrm{CDCl}_{3}\right) \delta 0.86-0.91(\mathrm{~m}, 3 \mathrm{H}), 1.18-1.32$ $(\mathrm{m}, 11 \mathrm{H}), 1.49-1.79(\mathrm{~m}, 1 \mathrm{H}), 1.95-2.13(\mathrm{~m}, 1 \mathrm{H}), 2.20-2.28(\mathrm{~m}, 1 \mathrm{H}), 2.44(\mathrm{~s}, 3 \mathrm{H}), 2.54-2.65(\mathrm{~m}$, $1 \mathrm{H}), 3.52-3.59(\mathrm{~m}, 1 \mathrm{H}), 4.13-4.22(\mathrm{~m}, 2 \mathrm{H}), 4.40-4.48(\mathrm{~m}, 1 \mathrm{H}), 7.33(\mathrm{~d}, J=8.4 \mathrm{~Hz}, 2 \mathrm{H}), 7.94(\mathrm{~d}, J$ $=8.4 \mathrm{~Hz}, 2 \mathrm{H}) ;{ }^{13} \mathrm{C} \mathrm{NMR}\left(75 \mathrm{MHz}, \mathrm{CDCl}_{3}\right) \delta 13.9,21.6,22.5,24.9,26.7,27.6,28.9,31.6,34.6$, $48.1,58.5,61.9,128.4,129.5,135.4,145.2,167.8,168.4$; EI-MS ( $m / z$, relative intensity): 396 $\left[(\mathrm{M}+1)^{+}, 5\right], 331$ (32), 310 (28), 258 (30), 155 (88), 91 (100). HRMS calcd for $\mathrm{C}_{20} \mathrm{H}_{29} \mathrm{NO}_{5} \mathrm{~S}$ : 395.1766, found: 395.1787; $\mathrm{C}_{20} \mathrm{H}_{30} \mathrm{NO}_{5} \mathrm{~S}:$ 396.1844, found: 396.1835. Anal. Calcd for $\mathrm{C}_{20} \mathrm{H}_{29} \mathrm{NO}_{5} \mathrm{~S}: \mathrm{C}, 60.76 ; \mathrm{H}, 7.39 ; \mathrm{N}, 3.54$. Found: C, 61.17; H, 7.37; N, 3.48.

(1) (a) Noguchi, Y.; Kurogi, K.; Sekioka, M.; Furukawa, M. Bull. Chem. Soc. Jpn. 1983, 56, 349-350. (b) Sas, W. J. Chem. Res. Synop. 1993, 160-161. 


\section{Diastereomeric mixtures}
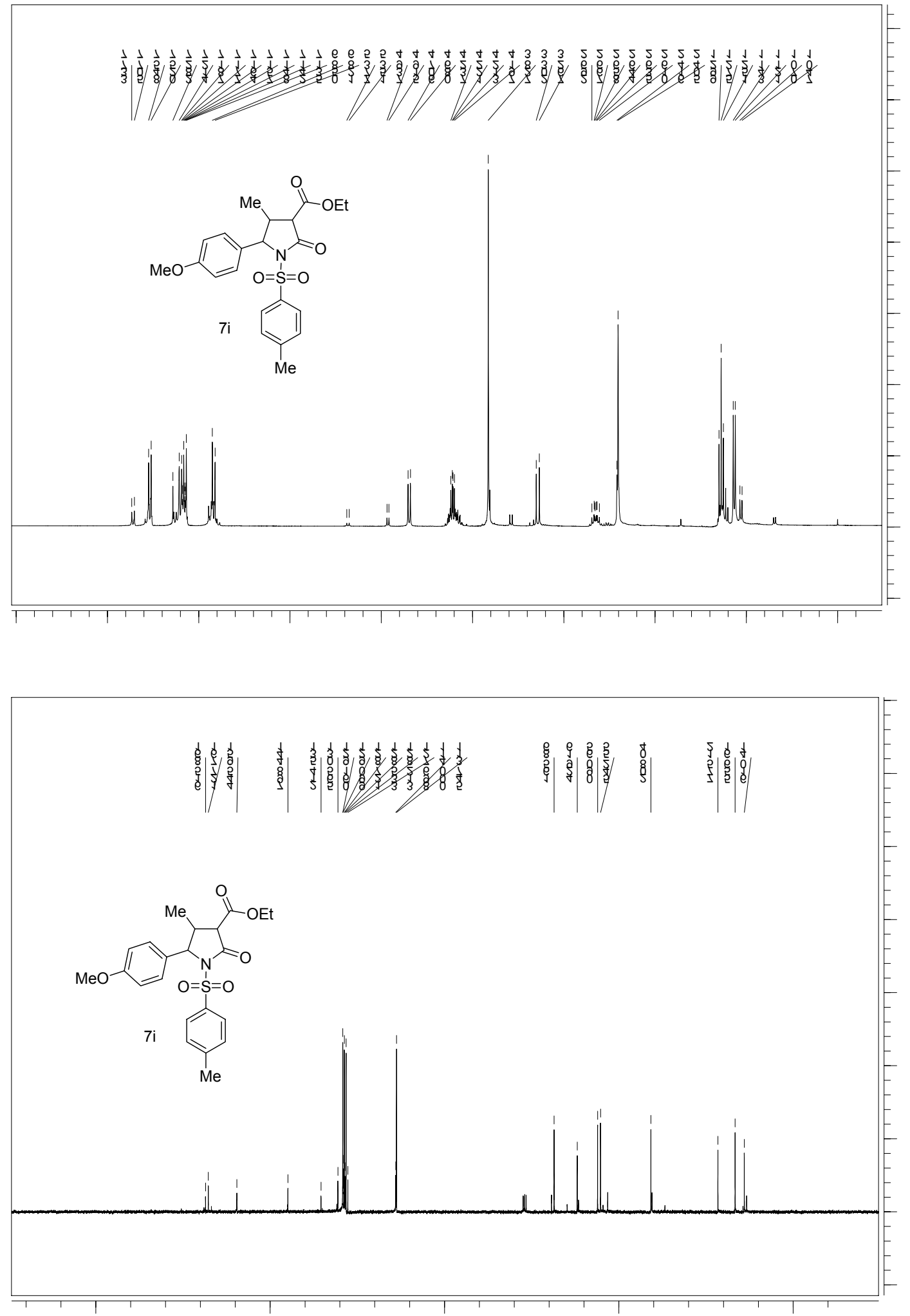

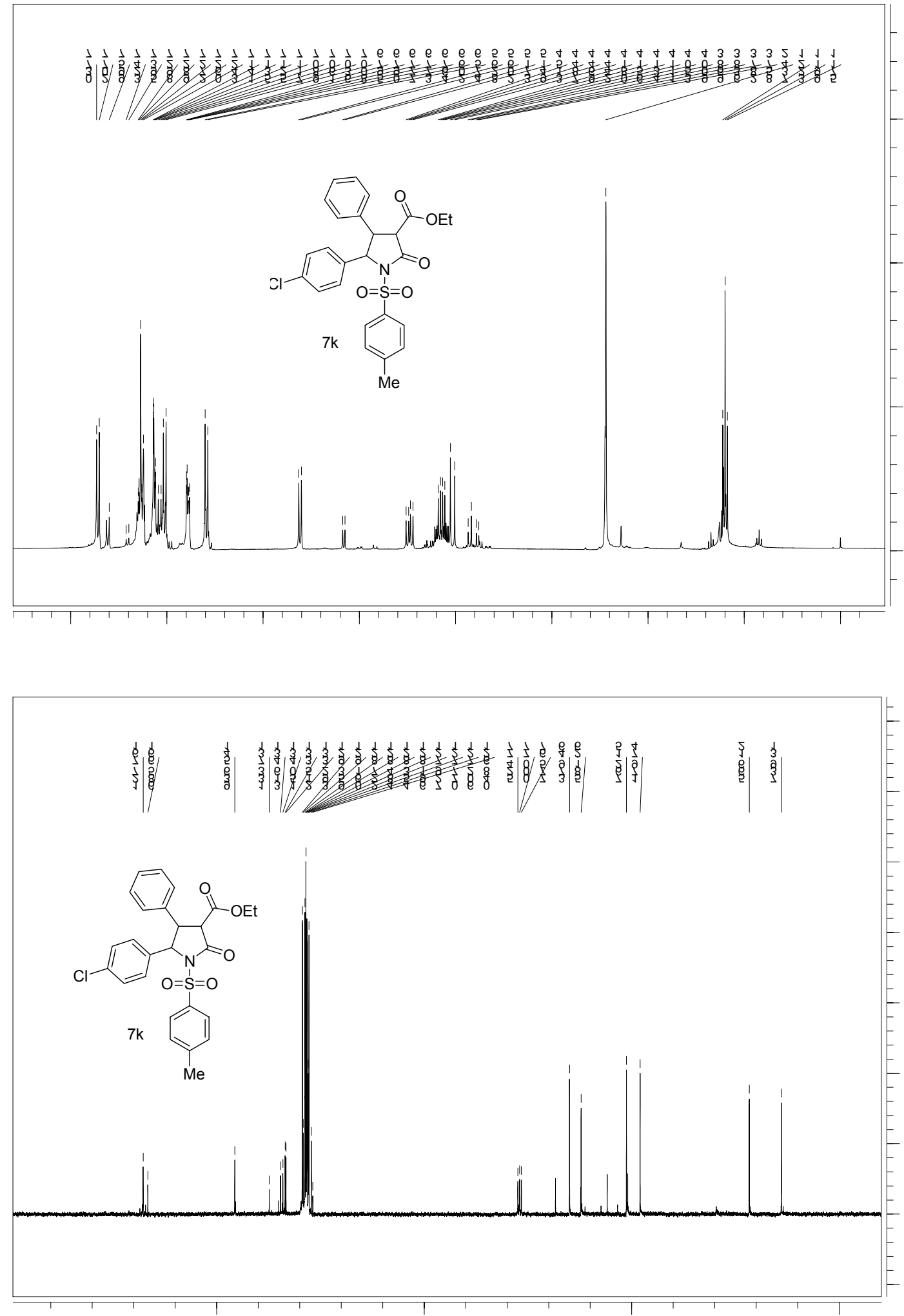


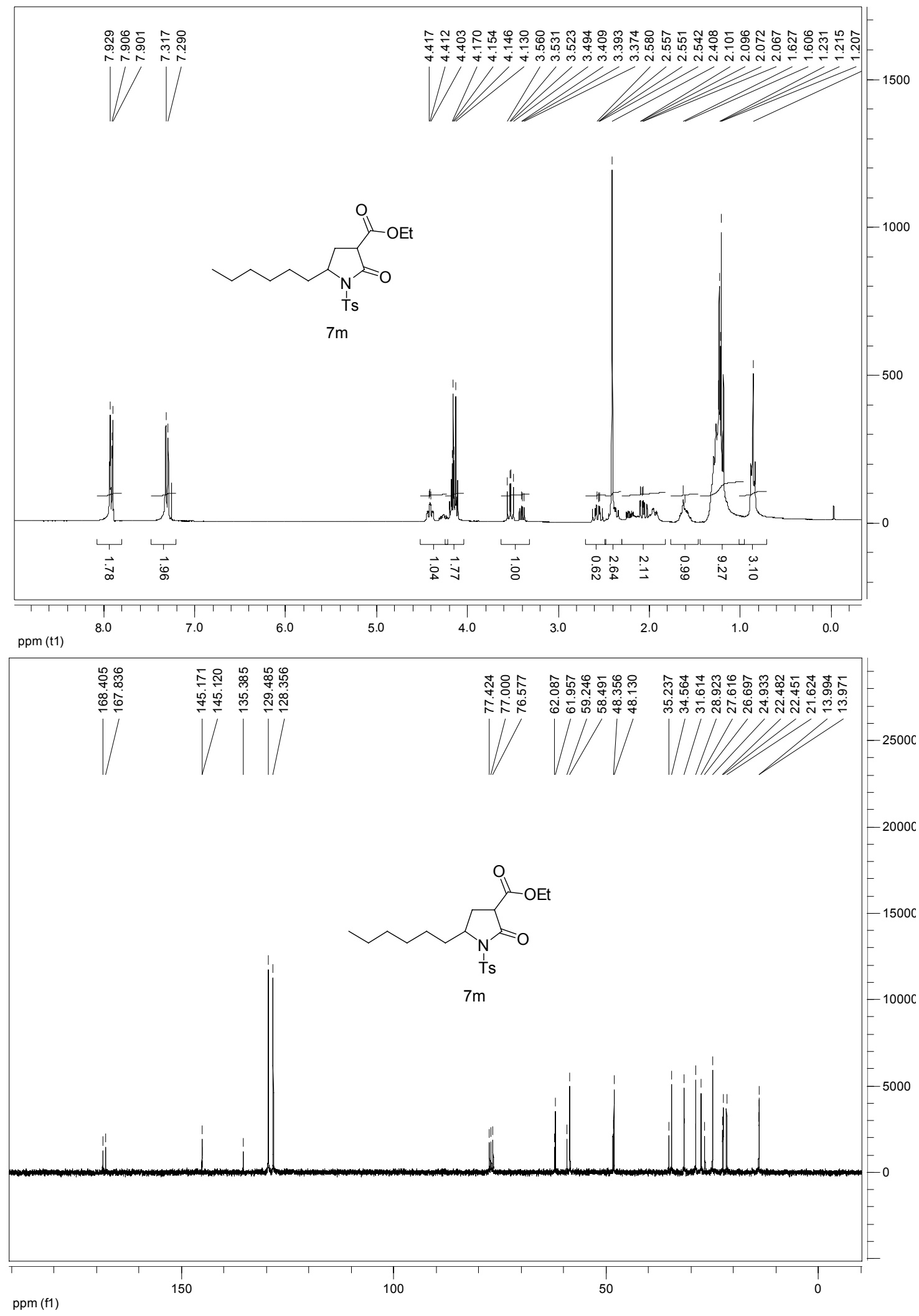

